\title{
European guidelines for quality assurance in colorectal cancer screening and diagnosis. First Edition Communication
}

Authors

Institutions
J. Austoker ${ }^{1}$, L. Giordano ${ }^{2}$, P. Hewitson ${ }^{1}$, P. Villain ${ }^{1}$

${ }^{1}$ University of Oxford, Oxford, United Kingdom

${ }^{2}$ CPO Piemonte, Turin, Italy
Key words

0 mass screening

colorectal neoplasms

- communication

- information strategies

- population-based programmes

- multidisciplinary evidencebased guidelines
Bibliography

DOI http://dx.doi.org/

10.1055/s-0032-1309809

Endoscopy 2012; 44:

SE164-SE185

(c) Georg Thieme Verlag KG

Stuttgart · New York

ISSN 0013-726X

Corresponding author

\section{P. Hewitson}

Department of Primary Health

Care

University of Oxford

Old Road, Headington

Oxford OX3 7LF

United Kingdom

paul.hewitson@dphpc.ox.ac.uk qas@iarc.fr
Multidisciplinary, evidence-based guidelines for quality assurance in colorectal cancer screening and diagnosis have been developed by experts in a project coordinated by the International Agency for Research on Cancer. The full guideline document covers the entire process of populationbased screening. It consists of 10 chapters and over 250 recommendations, graded according to the strength of the recommendation and the supporting evidence. The 450-page guidelines and the extensive evidence base have been published by the European Commission. The chapter on

\section{Background}

$\nabla$

According to the most recent estimates by the International Agency for Research on Cancer [30] colorectal cancer (CRC) is the most common cancer in Europe with 432000 new cases in men and women reported annually. It is the second most common cause of cancer deaths in Europe with 212000 deaths reported in 2008. Worldwide CRC ranks third in incidence and fourth in mortality with an estimated 1.2 million cases and 0.6 million deaths annually. The European Union (EU) recommends population-based screening for breast, cervical and colorectal cancer using evidence-based tests with quality assurance of the entire screening process including diagnosis and management of patients with screen-detected lesions [18]. The EU policy takes into account the principles of cancer screening developed by the World Health Organization [119] and the extensive experience in the EU in piloting and implementing population-based cancer screening programmes [115]. Screening is an important tool in cancer control in countries with a significant burden of CRC, provided the screening services are high quality [116]. The presently reported multidisciplinary, evidence-based guidelines for quality assurance in colorectal cancer screening and communication includes 35 graded recommendations. The content of the chapter is presented here to promote international discussion and collaboration by making the principles and standards recommended in the new EU Guidelines known to a wider professional and scientific community. Following these recommendations has the potential to enhance the control of colorectal cancer through improvement in the quality and effectiveness of screening programmes and services.

diagnosis have been developed by experts and published by the EU [97].

\section{Methods}

$\nabla$

The methods used are described in detail elsewhere in this supplement [73]. Briefly, a multidisciplinary group of authors and editors experienced in programme implementation and quality assurance in colorectal cancer screening and in guideline development collaborated with a literature group consisting of epidemiologists with special expertise in the field of CRC and in performing systematic literature reviews. The literature group systematically retrieved, evaluated and synthesized relevant publications according to defined clinical questions (modified PatientIntervention-Comparison-Outcome-Study method). Bibliographic searches for most clinical questions werelimited to the years 2000 to 2008 and were performed on Medline, and in many cases also on Embase and The Cochrane Library. Additional searches were conducted without date restrictions or starting before 2000 if the authors or editors who were experts in the field knew that there were relevant articles published before 2000. Articles of adequate quality recommended 
by authors because of their clinical relevance were also included. Only scientific publications in English, Italian, French and Spanish were included. Priority was given to recently published, systematic reviews or clinical guidelines. If systematic reviews of high methodological quality were retrieved, the search for primary studies was limited to those published after the last search date of the most recently published systematic review, i.e. if the systematic review had searched primary studies until February 2006, primary studies published after February 2006 were sought. If no systematic reviews were found, a search for primary studies published since 2000 was performed.

In selected cases references not identified by the above process were included in the evidence base, i.e. when authors of the chapters found relevant articles published after 2008 during the period when chapter manuscripts were drafted and revised prior to publication. The criteria for relevance were: articles concerning new and emerging technologies where the research grows rapidly, high-quality and updated systematic reviews, and large trials giving high contribution to the robustness of the results or allowing upgrading of the level of evidence.

The methodological quality of the retrieved publications was assessed using the criteria obtained from published and validated check lists. Evidence tables were prepared for the selected studies. The evidence tables, clinical questions and bibliographic literature searches are documented elsewhere [72].

In the full guidelines document prepared by the authors and editors [97] over 250 recommendations were formulated according to the level of the evidence and the strength of the recommendation using the following grading scales.

\section{Level of evidence:}

I multiple randomised controlled trials (RCTs)

of reasonable sample size, or systematic reviews (SRs) of RCTs

II one RCT of reasonable sample size, or 3 or less RCTs with small sample size

III prospective or retrospective cohort studies or SRs of cohort studies; diagnostic cross sectional accuracy studies

IV retrospective case-control studies or SRs of case-control studies, time-series analyses

V case series; before/after studies without control group, cross sectional surveys

VI expert opinion

\section{Strength of recommendation:}

A intervention strongly recommended for all patients or targeted individuals

B intervention recommended

C intervention to be considered but with uncertainty about its impact

D intervention not recommended

E intervention strongly not recommended

Some statements of advisory character considered to be good practice but not sufficiently important to warrant formal grading were included in the text.

\section{Results}

\section{$\nabla$}

Thirty-five graded recommendations are included in Chapter 10.

\section{Recommendations ${ }^{1}$}

10.1 Developing communication strategies for an organised CRC screening programme is important to ensure that as many of the target population as possible receive the relevant information to be able to make informed decisions about whether or not they wish to attend CRC screening (VI-A). Sect 10.2.2.2 $^{-1}$

10.2 Any framework developed to communicate CRC screening information must enable individuals to make an informed decision, and should be underpinned by the four ethical principles of autonomy, non-malfeasance, beneficence and justice ( $\mathrm{VI}-\mathrm{A})$. ${ }^{\text {Sect 10.2.2.2 }}$

10.3 CRC screening programmes should provide balanced, quantified and unbiased information about CRC (e.g. incidence, risk factors and symptoms) and CRC screening (benefits, harms and risk factors). Scientific evidence should be used to develop patient information materials and should be easily accessible for public consultation (VI-A). ${ }^{\text {Sect } 10.2 .2 .2}$

10.4 CRC screening programmes should identify the barriers, needs and facilitators to informed decision-making (IDM) of their target population (including specific groups) (VI-A). The information materials produced, including written instructions on how to use the FOBT kit or perform the bowel cleansing procedure, and the intervention(s) used must conform to these identified information needs and facilitators. The public should be involved in the entire process, from identifying barriers, needs and facilitators to developing information materials (VI-A). ${ }^{\text {Sect 10.2.2.2 }}$

10.5 To communicate CRC screening information, including written instructions on how to use the FOBT kit or perform the bowel cleansing procedure, the language and text format used should be easy to understand and illustrations may be used. Ideally, written information (including written instructions) should not be the only source of information and should be complemented by visual communication instruments and/or oral interventions (VI-A). Sect 10.2.2.2

10.6 Primary health care providers should be involved in the process of conveying information to people invited for

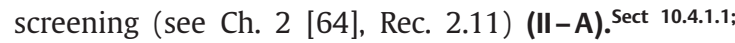
2.4.3.4; 2.4.3.4.1

10.7 In the context of an organised programme, personal invitation letters, preferably signed by the GP, should be used. A reminder letter should be mailed to all non-attenders to the initial invitation (see Ch. 2 [64] Rec. 2.8) (I-A). Sect 10.4.1.2; 2.4.3.4.1, 2.4.3.2

10.8 Although more effective than other modalities, phone reminders may not be cost-effective (see Ch. 2 [64], Rec. 2.9) (II-B). Sect 10.4.1.2; 2.4.3.2

\footnotetext{
1 Sect (superscript) after each recommendation in the list refers the reade to the section/s of the Guidelines dealing with the respective recommendation.*

* The first digit of the section numbers and recommendation numbers refers to the respective chapter in the guidelines. For Chapters 1 to 9 see: [57, $64,74,44,111,103,89,102,4]$ respectively.
} 
10.9 Mailing of the FOBT kit may be a good option, taking into account feasibility issues (such as reliability of the mailing system and test characteristics) as well as factors (such as the expected impact on participation rate) that might influence cost-effectiveness (see Ch. 2 [64], Rec. 2.15) (II-B). Sect 10.4.1.3; 2.5.1.1

10.10 clear and simple instruction sheets should be provided with the kit (see Ch. 2 [64], Rec. 2.16) (V-A). Sect 10.4.1.3; 2.5.1.1

10.11 Use of a non-tailored leaflet for the general population is advised; the leaflet should be included with the invitation letter. Information about CRC screening risks and benefits, CRC risks (incidence and risks factor), meaning of test results, potential diagnostic tests and potential treatment options should be included (VI-A). Illustrations may be used, which would be particularly useful for minorities, the elderly or low-literacy participants (II-A). sect 10.4.2.1 $^{\text {(II) }}$

10.12 A tailored leaflet for "harder to reach" groups could be used if these groups can be identified (II-B). Sect 10.4.2.1

10.13 Although there is good evidence that leaflets can increase knowledge of CRC screening, there is inconclusive evidence on the impact of leaflets on informed decision making (IDM). As a consequence, other interventions should be used in addition to leaflets (VI-A). . $^{\text {Sect 10.4.2.1 }}$

10.14 Video/DVD may be a useful component in a multi-modal intervention in addition to written information, and would be particularly useful for the elderly, minorities and low literacy participants (I-B). For the elderly, increasing the number of components of the multi-modal intervention and the period over which these components are provided may be more effective (I-B). ${ }^{\text {Sect }}$ 10.4.2.2.1

10.15 A computer-based decision aid could be used to help both the general population and specific groups to make informed decisions about CRC screening (I-B). The computer-based decision aid should be "userfriendly" and designed to fit with the computer abilities of the target population (general or specific groups). ${ }^{\text {sect }}$ 10.4.2.2.2

10.16 ICT-generated reminders ${ }^{2}$ to physicians could be used as an opportunity to provide counselling to patients on CRC and CRC screening, if primary care or other health practitioners are involved, and if patient medical records are electronic and give screening status $(\mathbf{I}-\mathbf{A}) .^{\text {sect }}$ 10.4.2.2.3

10.17 If possible, all information provided by the screening programme should be available on a specific web site. This information should be regularly updated (VI-A). Sect 10.4.2.2.4

10.18 It is not cost-effective or feasible to implement a tailored reminder telephone call in the general population. It may be possible for CRC screening programmes to use such an intervention for "harder to reach" groups if these groups can be identified (II-B). For example peer telephone support could be used. ${ }^{\text {Sect }}$ 10.4.2.3.1

10.19 Patient navigation could be used within CRC screening programmes, particularly to reach subgroups of the population such as the elderly, those with low literacy, and medically underserved patients. When used with minorities, the patient navigator should be from a sim- ilar ethnic background and/or live in the same community as the participant (I-B). Sect 10.4.2.3.2 $^{\text {10.3. }}$

10.20 Verbal face-to-face interventions with a nurse or physician could be used to improve knowledge and participation. They would be useful to reach subgroups of the population such as the elderly, minorities and those with low literacy (I-A). Sect 10.4.2.3.3

10.21 Nurses and primary care practitioners (GPs) should receive adequate training to be able to help people make informed decisions about CRC screening (VI-A). ${ }^{\text {Sect }}$ 10.4.2.3.3

10.22 Community-based verbal face-to-face interventions such as church-based sessions or in-person interviews could be used to reach minorities, in the case where the providers of such interventions received adequate training (II-B). Sect 10.4.2.3.3 $^{\text {10.3 }}$

10.23 Mass media campaigns using celebrities may be used to increase the awareness of CRC and CRC screening programmes. However these should be complemented by other measures as the effects are only temporary (V C). Sect 10.4.2.4

10.24 When addressed to minority groups, information provided by mass media campaigns should emphasise positive progress made by the minority group instead of emphasising racial disparities ( $\mathbf{V I}-\mathbf{C})$. . ect 10.4.2.4

10.25 CRC screening programmes should work closely with advocacy groups and the media and provide them with up-to-date, accurate and comprehensive information about CRC and CRC screening (VI-A). sect 10.4.2.4; 10.4.2.5

10.26 A telephone or ideally a verbal face-to-face intervention, e.g. nurse or physician intervention, should be used to inform a patient of a positive screening test result, as obtaining such a result could be a source of psychological distress for the patient. A letter informing the patient should not be used as the only way of notifying a positive result $(\mathrm{VI}-\mathbf{A})$. $^{\text {Sect } 10.4 .3}$

10.27 To increase endoscopy follow-up after a positive FOBT and facilitate communication, CRC screening programmes should, where possible:

- Use a reminder-feedback and an educational outreach intervention targeted to the primary care physician (II-A);

- Provide patients with a written copy of their screening report (II-A);

- Facilitate patient consultation with a gastroenterologist $\mathbf{( V - B )}$;

- Describe the follow-up procedure, make the followup testing more convenient and accessible (VI-A); and

- Use direct contact intervention to address psychological distress and other specific barriers. (V-B). Sect 10.4.3

10.28 Each endoscopy service must have a policy for pre-assessment that includes a minimum data set relevant to the procedure. There should be documentation and processes in place to support and monitor the policy (see

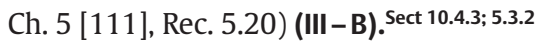

10.29 The endoscopy service must have policies that guide the consent process, including a policy on withdrawal of

\footnotetext{
2 ICT-generated reminders are produced electronically using information and communication technologies. ${ }^{*}$
} 
consent before or during the endoscopic procedure (see Ch. 5 [111], Rec. 5.25) (VI-B). Sect 10.4.3; 5.3.1

10.30 Before leaving the endoscopy unit, patients should be informed about the outcome of their procedure and given written information that supports a verbal explanation (see Ch. 5 [111], Rec. 5.26) (VI-A). Sect 10.4.3; 5.5.3

10.31 The outcome of screening examinations should be communicated to the primary care doctor (or equivalent) so that it becomes part of the core patient record (see Ch. 5 [111], Rec. 5.27) (VI-B). Sect 10.4.3; 5.5.5

10.32 Ideally, the invitation letter and the letter used for notification of a positive result should be sent with a leaflet and should encourage participants to read it ( $\mathbf{V I}-\mathbf{A})$. . $^{\text {Sect }}$ 10.5.1

10.33 Certain basic information, e.g. logistic/organisational information, description of the screening test, harms and benefits of screening, information about the FOBT kit and the bowel cleansing procedure, must be included in the invitation/result letter in case a person reads only the letter and not the leaflet ( $\mathbf{V I}-\mathbf{A})$. Sect 10.5.1

10.34 Recommendations when FOBT is used for screening: FOBT invitation letter, FOBT invitation leaflet, FOBT result/follow-up letter, see Section 10.5.2.

10.35 Recommendations when FS or colonoscopy (CS) is used for screening, either as primary screening test (FS or CS) or to follow-up a positive FOBT result (only CS): Endoscopy invitation letter, Colonoscopy leaflet, Endoscopy result/follow-up letter, see Section 10.5.3.

\subsection{Introduction}

7

10.1.1 Using communication strategies for a colorectal cancer screening programme: goals and challenges

The essential goal of colorectal cancer (CRC) screening programmes is to reduce illness and death due to colorectal cancer. This requires the need to ensure that as many of the target population as possible receive the relevant information to be able to make informed decisions about whether or not they wish to attend CRC screening. As adverse effects are intrinsic to screening practice, participants should understand that a balance exists between benefits and harms associated with CRC screening. In the policy brief Screening in Europe, [46] state that there is "above all, an imperative to involve participating individuals in decisions on screening and to give them clear and understandable information about what it involves". A key component of CRC screening programmes, therefore, is the information and education provided about CRC and CRC screening tests and procedures: people who use CRC screening services should receive accurate and accessible information that reflects the most current evidence about the CRC screening test and its potential contributions to reducing illness as well as information about its risks and limitations.

Providing effective information is particularly challenging in CRC screening. In contrast to other type of cancer screening, e.g. cervical or breast, CRC screening is indeed far more complex:

- There are multiple tests (FOBT, FS and Colonoscopy), which could be used for CRC screening, and information that should be given to the patient related to each of these tests is different;

- Some CRC screening tests (e.g. Colonoscopy or FS) are invasive and have known adverse effects; and
- Some CRC screening procedures (FOBT screening test and preparation for endoscopy screening (bowel cleansing procedure)) are generally undertaken without supervision from a healthcare professional; therefore specific instructions on how to use the FOBT kit or perform the bowel cleansing procedure need to be communicated to the patient.

This complexity may generate an additional source of anxiety for patients. Communication strategies that are used in other types of cancer screening programmes may not be suitable and/or sufficient to address both CRC screening complexity and this additional source of anxiety. Moreover the success of FOBT and endoscopy screening may rely on patient's understanding of the written instructions to perform the FOBT test or the bowel cleansing procedure; how this is communicated and then acted upon is crucial. Barriers that influence comprehension of written instructions (e.g. low literacy) could be a major issue in CRC screening.

\subsubsection{Purpose of this chapter}

There are two primary objectives of this chapter: First, to give people involved in providing and/or managing CRC screening (e.g. managers, decision-makers, health professionals etc.) an insight into the complexity of communication in CRC screening and its related critical issues; and second, to provide them with pragmatic recommendations on information strategies/tools/interventions that could be used. These recommendations mainly refer to an organised (and centralised) CRC screening programme, as this represents the gold standard to achieve (see Chapters 1 [57] and 2 [64]. In this communication chapter, we specifically provide guidance for FOBT screening programmes. Indeed, most of the EU countries are using FOBT as the primary screening test and more may adopt this test based on these EU guidelines recommendations (see Chapter 4 [44]). Most of the recommendations can be applied to endoscopy programmes as well.

\subsection{General principles}

\section{$\nabla$}

10.2.1 Informed decision-making, ethical principles

In the past few years, the autonomy of patients and their right to make informed decisions has become a central issue in medical interventions. Informed decision-making is a decision process in which individuals are supposed to make a rational and autonomous choice concerning their own health in order to protect themselves from risks and harms. It implies that these patients know the pros (benefits) and cons (harms) of screening and are aware not only of all the risks and benefits of participation in screening but also of non-participation $[5,40,90]$. Receiving information about the cancer itself seems also important in the informed decision-making process [50]. As a consequence, any framework developed to communicate health information about CRC screening needs to be underpinned by the following ethical principles [9]:

- Autonomy: the obligation to respect the decision-making capacities of autonomous persons. This obligation emphasises that patients should normally be in a position to choose whether to accept an intervention or not as part of their general right to determine their own lives;

- Non-malfeasance: the obligation to avoid causing harm intentionally or directly (the principle is not necessarily violated if a proper balance of benefits exists; that is, if the harm is not di- 
rectly intended, but is an unfortunate side-effect of attempts to improve a person's health);

- Beneficence: the obligation to provide benefits, balancing them against risks; and

- Justice: the obligation of fairness in the distribution of benefits and risks.

Provision of balanced, unbiased and quantified information about CRC (e.g. incidence, risk factors and symptoms) and CRC screening (benefits, harms and risk factors) is crucial for helping patients in making informed decisions. It is important that scientific evidence is used to develop patient information materials, and that this evidence is easily accessible for public consultation. For example, in the UK, the summary of the evidence used in the development of the NHS National Bowel Cancer Screening Programmes patient information materials (Bowel Cancer Screening: The Facts and Bowel Cancer Screening: The Colonoscopy Investigation) is available on the NHS Cancer Screening Programme Website: http://www.cancerscreening.nhs.uk/bowel/publications/nhsbcsp04.html.

\subsubsection{Identifying and reducing barriers/obstacles to informed decision making}

Informed decision-making (IDM) is a complex process. Receiving balanced, unbiased and quantified information related to CRC and CRC screening may be not sufficient for patients to make informed decisions; patients need also to be able to understand the information provided, to make a decision and to carry out their decision [84]. Barriers/obstacles to IDM may exist and may be related to:

- The setting and the organisation of the CRC screening programme, such as the access and the availability of the screening service and the access and the availability of the screening information (see Chapter 2 [64]);

- The knowledge, attitudes and practice of the CRC screening provider(s) (see Chapter 2 [64] and 10.4.2.3.3); or

- The patient themselves: age, gender [35], physical or mental health problems, occupation, education or abilities to read or understand information (see below) may be barriers to IDM. In some cases, risk information can be also a barrier [101,120].

It is important to understand what these barriers are so that measures can be taken to overcome them.

\subsubsection{Barriers related to the patients themselves \\ Population heterogeneity}

Health professionals offering screening to the population have to deal with individuals of different ages and with different cultures, values and beliefs. For these reasons, the information provided may be viewed differently and what is best for one recipient may not be the best for another [39,92]. In addition, contextual and personal factors may directly influence the way an individual processes health information and may therefore affect the motivations to attend screening. Educational status can also have an impact on how the presented information is understood $[3,21,55]$.

\section{Ethnic minorities}

Providers of screening programmes frequently have to cater to multicultural and multi-linguistic populations with all the related communication problems. Overcoming these problems requires more than just translating the information material. An understanding should be gained of ethno-cultural values, beliefs, health practices and communication styles of these varied groups, and the information materials produced must conform to these identified needs [113].

\section{Low health literacy}

Inadequate or low health literacy is defined as the inability to read and comprehend basic health-related information. Health literacy requires a complex group of reading, listening, analytical, and decision-making skills, and the ability to apply these skills to health situations. Low health literacy is independently linked to mortality and a range of poor health outcomes $[6,24,105,106]$. Poverty, ethnicity and age are also considered predictors of limited literacy [21]. In most countries, low literacy is a widespread problem as is low numeracy. In the UK $16 \%$ of the population (5.2 million adults) are classified as having lower literacy [100] and $47 \%$ (15 million adults) as having low numeracy. In a screening context, low health literacy can represent a major obstacle in understanding cancer screening information, diagnosis, treatments options, etc. This is particularly true in CRC screening as the demands of written information are perhaps greatest (see 10.1.1). In a group of US male veterans, those with low literacy were 3.5 times as likely not to have heard about colorectal cancer, 1.5 times as likely not to know about the FOBT screening test, and more likely to have negative attitudes about the FOBT [28]. Specifically, they were 2 times as likely to be worried that FOBT was "messy", and 4 times as likely to state that they would not use an FOBT kit if their physician recommended it.

In order to achieve health literacy, it is important that health and screening operators ascertain people's needs by using appropriate communication strategies, promoting access, identifying and removing barriers/obstacles within systems, and continuously evaluating the efforts to ensure improvement.

\subsubsection{Reducing barriers}

As there are many communication interventions that could be used ( Fig. 10.1 and section 10.4), CRC screening programmes should identify what would be the most appropriate communication strategy(ies) to use for their target population (including specific groups); CRC screening programmes should take into account their population barriers, needs and facilitators to IDM. The information materials produced must conform to these identified information needs and facilitators. The public perspective is important for appropriate understanding of these barriers, needs and facilitators. The public should be involved when communication tools are developed.

To reduce individuals' barriers, especially related to language and ways of processing information, CRC screening should provide information in a practical and concise way, using a simple and clear language, avoiding jargon and technical terms, such as incomprehensible mathematical or statistical concepts for expressing risk, and illustrations should be used (see also 10.4.2.1). This is particularly true for written instructions on how to use the FOBT kit or perform the bowel cleansing procedure.

Ideally, written information (including written instructions) should not be the only source of information and should be complemented by visual communication instruments and/or verbal interventions.

\section{Summary of evidence}

- Developing communication strategies in CRC screening programmes is important to ensure that as many of the target population as possible receive the relevant information to be able to make informed decisions about whether or not they wish to attend CRC screening.

- Providing effective communication is particularly challenging in CRC screening as CRC screening is far more complex than 


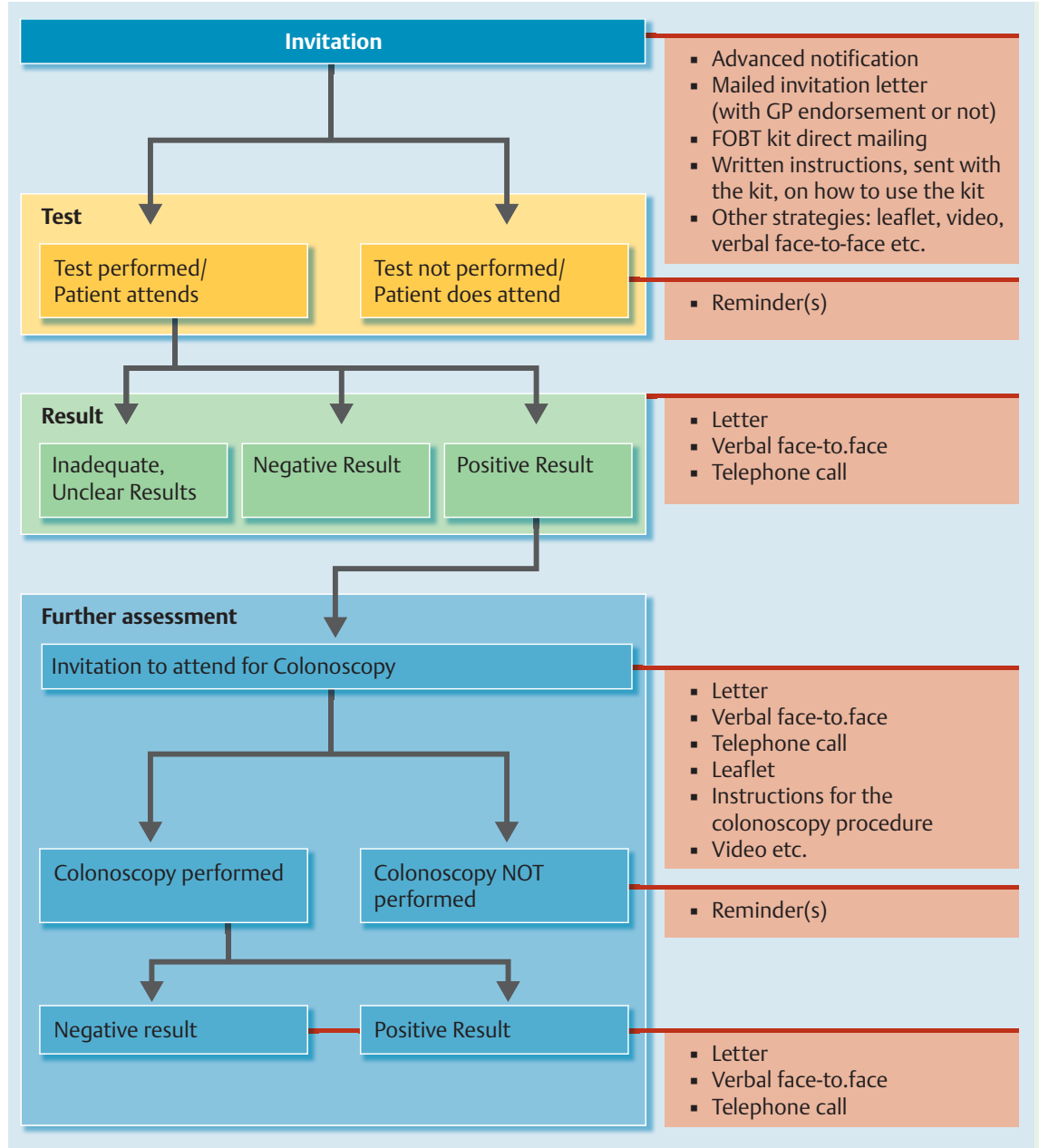

Fig. 10.1 Communication tools in FOBT-CRC screening.

(n)

(with GP endorsement or not)

ritten instructions, sent with

Other strategies: leaflet, video, verbal face-to-face etc.

other types of cancer screening. Communication strategies adopted/used in other types of cancer screening may not be suitable and/or sufficient to address CRC screening complexity and the additional source of anxiety generated for patients. Some screening procedures (e.g. FOBT) may rely on patient's understanding of the written instructions; how this is communicated and then acted upon is essential.

- Any framework developed to communicate CRC screening information must enable individuals to make an informed choice and should be underpinned by the four ethical principles of autonomy, non-maleficence, beneficence and justice. Informed decision making (IDM) in screening supposes that people make a rational and autonomous decision to participate, knowing the pros and cons of screening and being aware of all risks and benefits of their participation (VI).

- CRC programmes should provide balanced, unbiased and quantified information about CRC (e.g. incidence, risks factors and symptoms) and CRC screening (benefits, harms and risks). Scientific evidence should be used to develop patient information materials and should be easily accessible for public consultation.

- Barriers/obstacles to IDM may exist and may be related to the setting and the organisation of the CRC screening programme, the knowledge, attitudes and practice of the CRC screening provider(s) or the patient themselves.

- CRC screening programmes should identify the barriers, needs and facilitators to IDM of their target population (including specific groups) (VI). An understanding should be gained of ethno-cultural values, beliefs, health practices and communication styles of the varied groups of the target population. Research should be carried out to identify how to better communicate information to low literacy groups in the population. The information materials produced (including the written instructions on how to use the FOBT kit or perform the bowel cleansing procedure) and the intervention(s) used must conform to these identified information needs and facilitators. The public should be involved in the entire process, from identifying barriers, needs and facilitators to developing information materials.

- To reduce individuals' barriers, especially related to language and ways of processing information, the language and text format should be easy to understand and illustrations should be used. Ideally, written information should not be the only source of information and should be complemented by visual communication instruments and/or oral interventions. This is particularly true for written instructions on how to use the FOBT kit or perform the bowel cleansing procedure (VI).

\section{Recommendations}

10.1 Developing communication strategies for an organised CRC screening programme is important to ensure that as many of the target population as possible receive the relevant information to be able to make informed decisions about whether or not they wish to attend for CRC screening ( $\mathrm{VI}-\mathbf{A})$.

10.2 Any framework developed to communicate CRC screening information must enable subjects to make an informed decision 
and should be underpinned by the four ethical principles of autonomy, non-maleficence, beneficence and justice ( $\mathrm{VI}-\mathrm{A})$.

10.3 CRC screening programmes should provide balanced, quantified and unbiased information about CRC (e.g. incidence, risk factors and symptoms) and CRC screening (benefits, harms and risks). Scientific evidence should be used to develop patient information materials and should be easily accessible for public consultation ( $\mathrm{VI}-\mathrm{A})$.

10.4 CRC screening programmes should identify the barriers, needs and facilitators to informed decision making (IDM) of their target population (including specific groups) (VI-A). The information materials produced, including written instructions on how to use the FOBT kit or perform the bowel cleansing procedure, and the intervention(s) used must conform to these identified information needs and facilitators. The public should be involved in the entire process; from identifying barriers, needs and facilitators to developing information materials (VI-A).

10.5 To communicate CRC screening information, including written instructions on how to use the FOBT kit or perform the bowel cleansing procedure, the language and text format used should be easy to understand and illustrations may be used. Ideally, written information (including written instructions) should not be the only source of information and should be complemented by visual communication instruments and/or oral interventions (VI-A).

\subsection{Communication tools/interventions used in CRC screening programmes

Organised screening programmes generally have three distinct "communication" phases throughout the CRC screening process, where information (general or person-specific information) can be provided to participants. For a CRC FOBT screening programme, $\bullet$ Fig.10.1 illustrates these three phases and the corresponding communication tools:

I. The invitation phase: people are invited to participate in screening. Information for this screening phase is generally provided through invitation letters and leaflets. Written instructions on how to use the FOBT kit are usually provided with the kit;

II. The reporting results phase: people are notified of the results of their screening test. Information conveyed during this phase may be very sensitive and the communication tools must be carefully crafted to address the people's information needs;

III. The follow-up phase: only for people with a positive FOBT result who require further assessment (colonoscopy). Usually information about colonoscopy is notified at the same time as positive results. This phase also involves information about management of the colonoscopy procedure.

\subsection{Effectiveness of communication interventions in CRC screening \\ $\nabla$}

In this chapter, we review all the principal communication interventions that have been used or are being used in CRC screening and assess their effectiveness and limitations. Even though it would be useful to evaluate the effectiveness of an intervention in facilitating IDM, it would be very difficult: there is a lack of agreement about the definition of IDM, and validated measures do not exist $[33,50]$. As a result, the majority of studies use parti- cipation or uptake as the main outcome of interest to assess the effectiveness of a communication intervention.

\subsubsection{Interventions used to invite a person undergo}

the test

The interventions listed in this section (10.4.1) are closely associated with the organisation of the screening programme. Therefore, they have already been discussed in detail in Chapter 2 [64] and this discussion will not be repeated here. The Summary of evidence and Recommendations sections are the same as in Chapter 2.

\subsubsection{Physician/GP endorsement}

\section{Summary of evidence}

- The impact of information conveyed with the invitation is greater if the invitation is signed by an individual's physician. Involvement of GPs also shows a positive influence on the impact of more tailored and structured information methods (II).

\section{Recommendations}

10.6 Primary health care providers should be involved in the process of conveying information to people invited for screening (see Ch. 2 [64]; Rec. 2.11; Sect. 2.4.3.4 and 2.4.3.4.1) (II-A).

\subsubsection{Letters}

\section{Summary of evidence}

- A personalised letter signed by a general practitioner or by another trusted primary health care providers is more effective than an impersonal letter sent by a central screening centre (I).

- An advance notification letter may increase participation (II).

- Any kind of reminder is effective in increasing adherence, with telephone reminders being the most effective option, but also the most expensive (I).

\section{Recommendations}

10.7 In the context of an organised programme, personal invitation letters, preferably signed by a GP, should be used. A reminder letter should be mailed to all non-attenders to the initial invitation (see Ch. 2 [64]; Rec. 2.8; Sect. 2.4.3.4.1 and 2.4.3.2) (I-A). 10.8 Although more effective than other modalities, phone reminders may not be cost-effective (see Ch. 2 [64]; Rec. 2.9; Sect. 2.4.3.2) (II-B).

\subsubsection{FOBT: delivery of the kit and instruction sheet} Summary of evidence

- There is no evidence that the proportion of inadequate samples may be affected by the provider used to deliver the kit, as long as clear and simple instruction sheets are provided with the kit (II-V).

- The time required to reach the test provider represents a strong determinant of compliance (II).

- Sending the FOBT kit together with the invitation letter may be more effective than letter alone, but the cost-effectiveness of such strategy might be low (II).

\section{Recommendations}

10.9 Mailing of the FOBT kit could be a good option, but feasibility issues (such as reliability of the mailing system and test characteristics), as well as factors (such as the expected impact on participation rate) that may influence cost-effectiveness must be taken into account (see Ch. 2 [64]; Rec. 2.15; Sect 2.5.1.1) (II-B). 
10.10 Clear and simple instruction sheets should be provided with the kit (see Ch. 2 [64]; Rec. 2.16; Sect 2.5.1.1) (V-A).

10.4.2 Other interventions which can be used with the invitation: written, visual, face-to-face interventions 10.4.2.1 Leaflets and booklets

Leaflets are a key way for the organisers of screening programmes to communicate with the target population. The results of a recently published study, in which an information leaflet was provided in addition to the invitation letter, showed that CRC participation was significantly higher among patients who read both the leaflet and the letter compared to those who read just the letter [98].

Two RCTs have investigated the effectiveness of leaflets in increasing participation in CRC screening either by FOBT [45] or colonoscopy [23]:

I. Hart et al. [45] showed that leaflets significantly increased participation in men but not in women. According to the authors, one possible explanation was that women are generally better informed that men about the benefits of screening as they are targeted by breast and cervical screening programmes. Hence the participation rate for women is higher than for men.

II. Denberg et al. [23] showed that a leaflet mailed before a scheduled appointment increased adherence to screening colonoscopy among patients receiving referrals for the procedure.

Five studies assessed the content of leaflet:

I. One survey [112] was conducted to qualify the level of knowledge obtained by using a leaflet that provided information similar to that used in leaflets designed for other European screening trials. Although the leaflet was reported to be clear and readable, the information provided in it was not always well understood. The authors concluded that other educational options should be investigated in order to improve general knowledge of CRC screening in patients.

II. In another RCT, Trevena, Irwig \& Barratt [109] assessed the relative effectiveness of using a comprehensive "decision-aid (DA) booklet" (20-page leaflet) and a 2-page leaflet that contained minimal information about false-positives and followup, no quantification of outcomes, no graphs or pictures, and no personal worksheet or examples. The results showed that providing more information about FOBT screening contributed to increasing informed choice, defined by the authors as: knowledge, clear values and screening intention (decision). There was no noticeable effect on the screening uptake.

III. Adding explanatory illustrations to written material about the polyp-cancer process and the removal of polyps during FS, significantly increased knowledge and understanding [12].

IV. Robb et al.'s RCT [93] showed that using leaflets that gave information on CRC risk factors with or without information on colorectal screening by FOBT and FS was effective in increasing knowledge about the risk factors for CRC without increasing anxiety.

V. In an experimental pilot study, Lipkus et al. [61] assessed the effect of adding information about CRC risks (CRC incidence and risk factors) and CRC severity (treatment modalities for CRC and two testimonials of patients living with advanced $\mathrm{CRC}$ ) in a leaflet for FOBT screening. Whereas perception of CRC risks had no apparent effect, perception of CRC severity significantly increased intention to be screened.

Four studies have assessed the effect of using tailored/targeted leaflets/booklets:
I. Myers et al. [78] investigated the impact of targeted and tailored interventions in an RCT by testing the effect of a leaflet addressing personal barriers to screening in one urban primary care practice. The barriers to screening were identified through a baseline telephone survey involving the entire test population. The impact of the telephone contact on the survey results is not known. The authors reported no significant difference between the interventions.

II. Lipkus et al. [62] assessed the effect of adding tailored information about CRC risks to a leaflet aimed at members of a specific occupational group (carpenters) by adding a section highlighting occupational risk factors that increased their personal CRC risk. The study showed that adding tailored risk factor information affected neither risk perception nor screening uptake.

III. Marcus et al.'s RCT [65] investigated the impact of targeted and tailored interventions on CRC screening participation outside of a primary care setting. Tailored messages were derived from a baseline telephone survey. Three tailored conditions were tested and compared to a non-tailored intervention (a booklet): a single-tailored intervention (a 16-page tailored booklet), a multiple-tailored intervention (the tailored booklet plus tailored leaflets mailed out over a 12-month period) and a multiple-re-tailored intervention (as the latter except that subsequent leaflets were "re-tailored" based on follow-up interviews). Over a 14-month period, the multipletailored intervention was more effective than the non-tailored one, which could be explained by the "multiple" nature of the intervention. When comparing the two multiple interventions, there was no effect of using "re-tailored" material. When age stratification was used, a significant effect of the single-tailored intervention compared to the non-tailored booklet was observed for the younger participants (ages 50 59). The impact of the baseline telephone survey is not known.

IV. Wardle et al. [117] evaluated the effect of a leaflet specially designed for a "harder-to-reach" group of people identified in the screening arm of a FS trial. In addition to presenting basic information on CRC and screening, the booklet addressed psychological barriers to the FS test. The booklet was shown to decrease negative attitudes toward FS screening and increased screening attendance.

According to these studies, there is good evidence that leaflets can increase knowledge of CRC screening, but the evidence that leaflets facilitate the exercise of informed choice is less obvious. Fox's systematic review [33] came to the same conclusions. As there is a lack of agreement about the definition of "informed choice" and validated measures [33,50], it is indeed difficult to evaluate the impact of leaflets use on patients' informed choice about CRC screening. Therefore, other interventions should be used in addition to leaflets.

\section{Summary of evidence}

- Non-tailored leaflets are effective in increasing screening participation and/or knowledge. Leaflets in addition to the invitation letter are valuable tools (I).

- Including more detailed information in a leaflet (e.g. information about false-positive and follow-up, quantification of outcomes, graphs and pictures, personal worksheets or examples) contributed to an increase in knowledge, clear values and screening intention (decision) but not uptake (I). 
- Providing information about risk factors for CRC was effective in increasing knowledge about the risk factors for CRC without increasing anxiety. Perception of CRC risks did not affect the uptake rate for FOBT screening (I).

- Adding illustrations to written material about the polyp-cancer process and the removal of the polyps during FS significantly increased knowledge and understanding (II).

- Tailored leaflets for "harder-to-reach" groups seem to be effective in increasing screening participation and knowledge (II).

- A tailored booklet compared to a non-tailored one proved more effective in increasing participation of younger participants. A multiple-tailored intervention over a period of time was more effective than using a non-tailored booklet (II). However, the impact of the baseline telephone survey to tailor the materials in this study cannot be evaluated.

- When using multiple-tailored interventions, there was no effect of using "re-tailored" material (II).

- It is difficult to prove that leaflets facilitate the exercise of IDM (I).

\section{Recommendations}

10.11 Use of a non-tailored leaflet for the general population is advised; the leaflet should be included with the invitation letter. Information about CRC screening risks and benefits, CRC risks (incidence and risk factors), meaning of test results, potential diagnostic tests and potential treatment options should be included (VI-A). Illustrations may be used, which would be particularly useful for minorities, elderly or low-literacy participants (II-A).

10.12 A tailored leaflet for "harder-to-reach" groups could be used if these groups can be identified. (II-B).

10.13 Although there is good evidence that leaflets can increase knowledge of CRC screening, there is inconclusive evidence on the impact of leaflets on informed decision making (IDM). As a consequence, other interventions should be used in addition to leaflets (VI-A).

\subsubsection{Videotapes/DVDs, interactive computer-} based decision aids, ICTs (information \& communication technologies) and Internet

\subsection{Videotapes/DVDs}

\section{a. Non multi-modal intervention}

Two US studies $[36,122]$ showed that using a videotape had no effect on the overall rate of CRC screening. In the second study the video, mailed before a scheduled examination, only modestly improved sigmoidoscopy screening rates.

Two studies by Griffith et al. [42] investigated the effect of introducing differential content in a DVD. In the first study, the DVD presented to both groups differed only in the inclusion of a segment where an individual discussed why he did not participate in screening. In the second study, two forms of a DVD were evaluated: one included two screening test options, and the other five screening test options. Participants' interest in CRC screening was investigated; neither study found a difference between the interventions.

Meade, McKinney \& Barnas [68] investigated whether a booklet or a videotape, both tailored to the target population of participants, was more effective for improving CRC knowledge, which was evaluated just after the intervention. Results indicated that both booklet and videotape significantly increased knowledge and there were no statistically significant differences between the 2 interventions, regardless of the patients' literacy levels. The "tailored" aspect of both of the interventions was one hypothesis to explain the absence of discrepancy between the two interventions.

\section{b. Multi-modal intervention including videotape/ DVD and print material}

Four studies $[14,59,87,88]$ assessed the effect of using a multimodal intervention, which included a videotape and print material:

I. Pignone et al.'s [87] RCT trial used an educational videotape, targeted brochure and chart marker. The study showed that the intervention, compared to no intervention, increased CRC screening participation.

II. In Lewis et al.'s [59] controlled trial the intervention consisted of a mailed package containing an educational videotape, a reminder letter from their physician, surveys to be completed before and after the video watching, and system changes allowing patients direct access to schedule screening tests. The study showed that the intervention, compared to no intervention, increased CRC screening participation.

III. Campbell et al.'s [14] randomised trial compared the effect of a tailored print and video intervention (4 personalised computer-tailored newsletters and videotapes), designed to target a rural minority (African-American) community, to a lay health advisor (a trained member of the community) intervention. The study showed that the tailored print and video intervention was more effective in increasing FOBT screening than no intervention. The authors reported suboptimal advisor reach and diffusion.

IV. Powe, Ntekop \& Barron [88] showed that a 5-phase culturally relevant intervention (video, calendar, poster, brochure, flier) among community elders and delivered over a 12-month period, significantly increased knowledge and screening participation compared to either a 6-month and 3-phase intervention or a single intervention (video or usual care). However, it is not possible to determine which aspects of the multi-modal intervention were most effective.

\section{Summary of evidence}

- A DVD alone had no effect on screening rates or interest in screening. Changing the video content did not affect this result. No difference was found between a tailored booklet and a tailored DVD regardless of the patients' literacy levels (I).

- When a video/DVD was used in a multi-modal intervention, an improvement in knowledge and increase in screening rates was observed. When the components of the multi-modal interventions were provided successively over a period of time, increasing the number of components and the period over which they were provided, there was an increased in knowledge and in participation of elderly people (I).

\section{Recommendations}

10.14 Video/DVD may be a useful component in a multi-modal intervention in addition to written information and would be particularly useful for the elderly, minorities and low literacy participants (I-B). For the elderly, increasing the number of components of the multi-modal intervention and the period over which these components are provided may be more effective (I-B). 
10.4.2.2.2 Interactive computer-based decision aids

Four studies $[27,54,69,71]$ showed that a computer-based decision aid improved patients' knowledge about screening and was useful to most in making decisions about screening (increased intention to be screened and increased interest in screening). The same results were obtained in rural primary care practices [38] and in a Hispano/Latino community [63] for which the decision aid was specifically designed.

Three studies have assessed the effect of a computer-based decision aid on screening participation:

I. An RCT by Ruffin et al. [95] showed that an interactive programme to help to establish a preference among the CRC screening tests options was more effective than an existing CRC website selected to represent the standard, state-of-the art and non interactive website.

II. In an uncontrolled trial, Kim et al. [54] tested the effect of an interactive computer-based decision aid including an audio track playing during the entire programme and explaining all of the figures that were presented, making the content accessible to users with varying levels of literacy. The intervention improved screening uptake.

III. Dolan and Frisina's [27] RCT showed that a computer-based decision aid designed to help patients choose between different strategies for CRC screening and including the option of 'no screening', when added to a simple educational interview intervention, had no effect on CRC screening uptake.

Jerant et al. [51] conducted an RCT comparing the effects of using a tailored versus a non-tailored interactive multimedia program. Besides a tailored component (e.g. specific screening recommendation tailored to the individual), the tailored programme also contained brief patients and physician video clips that were not in the non-tailored intervention. The study showed that the tailored programme was significantly more effective in bolstering CRC screening readiness and self-efficacy than the non-tailored intervention. It is not clear to what extent the video clips component of the tailored computer-based decision aid contributed to the result.

\section{Summary of evidence}

- Interactive computer-based decision aids improved knowledge and were useful in helping people decide whether or not to be screened. The same results were obtained in rural primary care practices and in an ethnic community for which the decision aid was specifically designed (I).

- Interactive computer-based decision aids increased screening participation, but had no effect if added to an interview intervention. A tailored computer-based intervention affected knowledge and intention to be screened more than a non-tailored intervention, but it is not clear to what extent the video clips component of the tailored computer-based decision aid contributed to the result (II).

\section{Recommendations}

10.15 A computer-based decision aid could be used to help both the general population and specific groups to make informed decisions about CRC screening (I-B). The computer-based decision aid should be "user-friendly" and designed to fit with the computer abilities of the target population (general or specific groups).
10.4.2.2.3 Information and communication technologies: future promises and challenges for enhancing CRC screening delivery Information and communication technologies (ICTs) are a diverse set of technological tools and resources used to communicate, create, disseminate, store, and manage information. ICT is sometimes referred to as simply Information Technologies (IT). ICTs include computers, the Internet, broadcasting technologies (radio and television), and telephones. They are typically used in combination rather than singly.

The European Union's Commission for Information Society and Media has defined eHealth as ICT-based tools covering "the interaction between patients and health-service providers, institution-to-institution transmission of data, or peer-to-peer communication between patients and/or health professionals" (http://ec. europa.eu/information_society/activities/health/whatis_ehealth/index_en.htm). Examples include health information networks, electronic health records, telemedicine services, wearable and portable systems which communicate, health portals, and many other ICT-based tools assisting disease prevention, diagnosis, treatment, health monitoring and lifestyle management. According to a recent systematic review [52], the published research using ICT in the context of cancer screening in general and CRC screening in particular almost exclusively tested the impact of ICT-generated reminders to either the provider alone or to both the patient and the provider. Dexheimer et al.'s review [25], found that ICT tools used to generate reminders, were either "computer-generated" (ICT tools were used to identify eligible patients and were integrated with electronic appointment systems so that reminders were automatically printed in advance of patient appointments and placed in the patient's chart) or "computerized" (ICT were used to identify eligible patient and generate electronic prompt).

There is ample evidence that patient- and provider-directed computerised reminder systems increase adherence in other cancer screening fields e.g. mammography. For CRC screening, three out of four recent studies showed that ICT-generated reminders to physicians increased CRC screening:

I. Sequist et al. [99] used computerized reminders, in both a passive and active form, added within each patient's electronic medical record, and thus visible by their physician during the appointment. Results showed that electronic reminders tended to increase screening rates among patients with 3 or more primary care visits.

II. Chan \& Vernon [15] tested the feasibility of using the NetLET website interface to provide patients with a personalised reminder from their physician to undergo CRC screening. The study concluded that it was not feasible to implement the NetLET. For the authors the lack of success was essentially due to the e-mail access barrier (patients without email at home or work) and the ICT system barrier itself, i.e. the complexity of accessing the NetLET website.

III. Nease et al. [82] investigated the effect of a computer-generated reminder placed in the patient's chart. The study showed that 11 out of 12 practices significantly increased their CRC screening rates and there was no significant difference between sending reminders either to clinician alone or to both patient and clinician.

IV. Jimbo et al.'s review [52] identified 13 studies evaluating the effect on ICT-generated reminders in FOBT CRC screening: 8 out of 13 studies showed that reminders increased FOBT screening participation. According to the EU commission (Information Society and Media), the widespread implementa- 
tion of ICT in health will increase the quality of healthcare services and will provide:

- Better information for patients and healthcare professionals;

- More efficient organisation of resources; and

- More "patient-friendly" healthcare services by helping

healthcare providers to be more flexible and better able to address the differing needs of individual patients.

Still "poverty and illiteracy in developing nations are major barriers to the adoption and sustainability of information technologies" [1]. Nevertheless, the existence of many successful implementations of ICT-enabled health communications and electronic health record systems in less industrialised countries in Africa [1], suggests that it is possible to bypass these barriers.

For [114], ICT is one of the "Six elements of a New Model of Primary healthcare delivery" in colorectal cancer screening. ICT use for interventions in screening in general, and in CRC screening more specifically, has the potential to go beyond simple reminder systems $[52,114]$. But to widely realise the potential of the use of IT in screening, patients' charts must provide the infrastructure to do this. Patients' charts must be organized enough to determine patient screening status and ideally physicians and clinics should use electronic medical records. According to Vernon \& Meissner [114] and Dexheimer et al. [25], these are areas that clearly need to be improved.

\section{Summary of evidence}

- ICT-generated reminders to physicians increased CRC screening rates (I). ICT has an important role to play in increasing efficiency of CRC screening and has the potential to go beyond simple reminder systems, and will provide better information for patients and healthcare professionals, more efficient organisation of resources and more "patient-friendly" healthcare services by providing a more flexible and personalised approach (I).

- To widely realise the potential of the use of IT in screening, patients' medical records should be improved to easily determine patient screening status, and ideally should be electronic (I).

\section{Recommendations}

10.16 ICT-generated reminders to physicians could be used as an opportunity to provide counselling to patients on CRC and CRC screening, if primary care or other health practitioners are involved, and if patient medical records are electronic and give screening status (I-A).

\subsubsection{Internet}

There is no evidence of the impact of the internet on screening in general and more specifically on CRC screening. Based on Della et al.'s review [22], the popularity of the internet as a conduit for health information is increasing. Still, not everyone is online; research indicates that higher usage of the internet is associated with younger age, more education and higher income $[11,22$, $34,86]$. As the variety of health information on the internet is expanding, source credibility continues to be a pivotal factor in determining the quality of information [22]. James et al. [48] performed a study of information seeking by cancer patients and their caregivers. This study has shown that "those who accessed Internet information, either directly or indirectly, reported high levels of satisfaction with it and generally rated it more highly than booklets or leaflets". The authors concluded that "the internet is an effective means of information provision in those who use it. Facilitated internet access and directed use by health professionals would be effective way of broadening access to this medium."

\section{Summary of evidence}

- There is no evidence of the impact of the Internet on CRC screening (VI).

- The popularity of the Internet as a conduit for health information is increasing (VI).

- People with younger age, more education and higher income have higher usage of the Internet (V).

- Source credibility continues to be a pivotal factor in determining the quality of information (V).

- Generally, using the internet as a source of information about cancer is more satisfying than leaflets or booklets (VI).

\section{Recommendations}

10.17 If possible, all information provided by the screening programme should be available on a specific web site. This information should be regularly updated (VI-A).

\subsubsection{Telephone intervention, patient navigator (PN)} intervention, and verbal face-to-face intervention other than PN

\subsection{Telephone intervention}

The majority of the studies assessed the impact of a reminder tailored telephone call added to printed materials (the "usual care"), which were incrementally added. In some studies, the intervention also included a booklet/leaftlet/brochure sent before the call. We retrieved seven studies:

I. Turner et al.'s RCT [110] compared a phone call by a trained peer coach with a mailed colonoscopy brochure about CRC screening in improving adherence to a first scheduled colonoscopy. Seven trained older patients who had had a colonoscopy served as peer coaches. The calls (1 per patient) were scheduled within two weeks of the colonoscopy appointment to address barriers to attendance. In this study peer coach telephone support significantly increased colonoscopy attendance. The fact that coaches received payment for each completed patient call might have introduced a bias in the study.

II. In Braun et al.'s RCT [10], the number of telephone calls has been suggested to have a negative effect on screening. The authors compared an intervention (one culturally targeted educational presentation) delivered by a nurse to an intervention delivered by physician and a peer, both of the same community background as the participants. The first intervention also included one reminder call, whereas the second intervention included multiple reminder telephone calls to encourage screening and address barriers. The two interventions realized similar gains in CRC knowledge but the education provided by the nurse was more effective in increasing uptake of CRC screening; one hypothesis to explain this result was that the multiple reminder phone calls made the intervention too invasive and burdensome.

III. Lairson's RCT [56] compared a usual care intervention (invitation letter, FOBT test, booklet and reminder letter) to tailored interventions, which incrementally added a tailored leaflet (two message pages) and a reminder telephone call to 
the usual care intervention. The most effective intervention was the intervention that used the tailored leaflet and the tailored telephone call reminder. An economic analysis showed that it was also the most costly.

IV. Three RCTs were performed either in a primary care population [17], at worksites for automobile industry employees [108], or in an HMO association [76]. These studies compared standard intervention to an intervention including printed materials along with tailored telephone outreach. In Costanza's RCT, the intervention did not increase colorectal cancer screening compared to control. In Tilley's RCT, the authors concluded that the tailored intervention (mailed invitation, tailored booklet followed by a tailored telephone call) produced a modest but higher screening participation compared to standard intervention (personal letters and flyers at the worksites). In Myers et al.'s survey (1994), adding to the control intervention (a FOBT kit and a reminder letter) a brochure followed by a phone call increased participation comparing to the control intervention.

V. Myers et al. [77] tested the effect of using usual care (i.e. mailing an advance letter, FOBT kit and a reminder letter) followed either by one telephone call intervention or by two calls plus a brochure intervention. The telephone outreach was used to resolve patient's barriers to non adherence or answer patientspecific questions. The study showed that one call significantly increased the participation compared to usual care. Moreover two calls seemed to have more impact than one on the participation rate.

Even if a tailored telephone call intervention seemed to be effective, it could certainly not be applicable as part of the normal invitation process in CRC screening for reasons of cost-effectiveness and the high volume of calls to be processed. It may be possible to implement tailored telephone calls for harder-to-reach groups if these groups can be identified.

\section{Summary of evidence}

- The majority of the studies assessed the impact of tailored reminder telephone call on CRC screening participation.

- A tailored telephone intervention seemed to be effective in increasing screening participation when used as a reminder to mailed invitation materials (usually booklet, FOBT kit, and mailed letter). The most effective but also the most costly intervention was to add to usual care a tailored leaflet and a tailored telephone call reminder.

Tailored telephone calls could certainly not be applicable as part of the normal invitation process for CRC screening for reasons of cost-effectiveness and the high volume of calls to be processed. It may be possible to implement tailored telephone call for "harderto-reach" groups if these groups can be identified (II-B). For example, peer coach telephone support for explaining colonoscopy procedure seemed to improve attendance for colonoscopy (II). It has been suggested that multiple reminder phone calls could make the intervention too invasive and burdensome.

\section{Recommendations}

10.18 It is not cost-effective or feasible to implement a tailored reminder telephone call in the general population. It may be possible for CRC screening programmes to use such an intervention for harder-to-reach groups if these groups can be identified (IIB). For example peer telephone support could be used especially to decrease the attendance barrier to colonoscopy (II-B). Multiple telephone calls seem to have more effect, but it is important to avoid coercion $(\mathbf{I}-\mathrm{C})$.

\subsection{Patient navigation/patient navigator}

A patient navigator ( $\mathrm{PN}$ ) is an individual whose role has been described as providing individualized assistance (by telephone and/ or by direct contact) to a patient to both educate and help them overcome healthcare system barriers related to, for example, doctors' offices, clinics, hospitals, out-patient centres, payment systems. In cancer screening, patient navigation should be considered as a method for guiding individuals through the cancer screening process [75]. "The client navigator approach included the traditional method (i.e. educated patients about cancer screening) along with a social worker who 'navigated' the health care system" [49]. By being able to provide social and logistical services, PN intervention should be differentiated from the usual "telephone intervention" (above section) or "verbal face-to-face intervention" (next section). Social and logistical services provided by patient navigators could be for example facilitating communication among patients/family members/survivors/healthcare providers, coordinating care among providers, facilitating appointments and follow-up appointments, and facilitating access and transportation to services facilities. Patient navigators could be trained community health workers/advisors who have close ties to the local community or trained social workers/health professional/volunteers or belong to a specific organization. The American Cancer Society (ACS) Patient Navigator Program, launched in 2005, currently operates in 60 sites across the USA. The ACS navigators are concentrated in hospitals and clinics that treat a large number of medically underserved patients.

\section{Summary of evidence}

- We retrieved eight recent US studies that examined the impact of involving PN in CRC screening in either urban public hospitals setting [75] or minority/ethnic urban community health centres $[7,16,26,49,58,80,85]$. In the minority/ethnic community, the PN was from a similar ethnic background and/or lived in the community from which the participants were recruited. Patient navigator intervention significantly increased the screening participation. The results of Myers et al.'s pilot study [75] are currently being tested in two RCTs.

\section{Recommendations}

10.19 Patient navigation could be used within CRC screening programmes, particularly to reach subgroups of the population such as the elderly, those with low literacy, and medically underserved patients. When used with minorities, the PN should be from a similar ethnic background and/or live in the same community as the participant (I-B).

10.4.2.3.3 Verbal face-to-face intervention other than PN: verbal face-to-face with GP, nurse or other health or trained non-health professional

As assessed by Wee et al.'s study [118], and other studies detailed in Chapter 2 [64], primary care physician (GP) counselling of patients has been positively associated with increasing CRC screening participation rates.

We retrieved eight studies that assessed the impact of direct interaction other than GP (e.g. face-to-face with nurse or other health or trained non-health professional) with participants either in the general population or in some specific subgroups of 
the general population, such as the socio-economically disadvantaged and/or belonging to racial/ethnic minority groups.

\section{a. In the general population}

Two studies $[104,107]$ evaluated the effect of one-to-one/faceto-face education about the FOBT screening process (purpose/ technique of obtaining samples/further testing) provided by a nurse and showed that the intervention increased the return rate of FOBT kits. Stokamer et al. (2005) also reported that participants in the intervention group were significantly less likely to contact the clinic with additional questions. In the study by Thompson et al., the nurse was also allowed to order FOBT kits that were given to patients before they left the clinic. This study showed an increased number of ordered kits.

Courtier et al. [19], evaluated the impact of a trained, non-healthcare professional who provided in-home information and a FOBT kit and personally collected the specimens from the participant's home. The study showed that CRC screening participation was higher in the intervention group.

In Hudson's study [47], practices that reported using nursing or health educator staff to provide behavioural counselling to patients on topics such as diet, exercise or tobacco also resulted in significantly increased CRC screening rates.

\section{b. In some specific sub groups of the general population}

Ford et al.'s RCT [32] tested different combinations of mail, reminder mail and call, phone call and in person church-based recruitment to invite older ( 55 - 74 years) African-American men in the Prostate, Lung, Colorectal and Ovarian (PLCO) cancer screening trial. They concluded that the most intensive intervention increased significantly the participation compared with the control or the other interventions. The most intensive intervention was the one that besides mail, telephone call, and reminder telephone call, added a face-to-face contact with participants (one session held at church).

Katz et al. [53] showed in a non-randomised trial that a community-based intervention (a face-to-face interview delivered by trained volunteers from the communities) performed among low-income women (78\% African-American) led to a significant increase in positive beliefs about CRC screening and in the intention to complete CRC screening in the next 12 months after the intervention. However CRC screening rates were not significantly increased 1 year after the intervention.

Based on Gren et al.'s paper [41], the American PLCO (The Prostate, Lung, Colorectal and Ovarian Cancer) screening trial of centres with enhanced minority recruitment programmes, relied extensively on community outreach, particularly church-based recruitment and in-person information sessions, to meet their goals.

\section{c. Quality of counselling}

In an observational study Ling et al. [60] evaluated a provider's (physician and nurse practitioner) intervention about CRC screening. They coded each intervention for nine elements of communication (Informed Decision-Making (IDM) Model) that have been shown to be important for IDM. The study showed that 6 of the 9 elements occurred in $\leq 20 \%$ of the visits with none addressed in $\geq 50 \%$. In this study, compared to patients whose understanding was not assessed, patients whose understanding was assessed during the visit had a higher rate of completing CRC screening. On the contrary, CRC screening participation was less when "patient's screening test preference" or "pros and cons of the alternatives" was discussed.

Ferreira et al.'s RCT [31] assessed the effect of trying to improve healthcare providers' (nurse practitioner and residents) counselling by using an intervention directed to the health-care provider. The intervention was a series of workshops on rationale and guidelines for CRC screening, and on strategies for improving communication with patients with low literacy skills. During the study, the healthcare providers received confidential information on their individual recommendation and adherence rates. The intervention significantly increased both recommendations and CRC screening completion (FOBT, endoscopy) among patients. The intervention also increased the screening rates among patients with low literacy skills.

\section{Summary of evidence}

- Verbal face-to-face intervention and education (nurse and GP) were clearly useful in improving knowledge and participation in CRC screening (I).

- A trained non-health professional, who provided in-home information and a FOBT kit and personally collected the specimens from the participant's home, was effective in increasing CRC screening (II).

- Practices, that reported using nursing or health educator staff to provide behavioural counselling to patients on topics such as diet, exercise or tobacco, also resulted in significantly increased CRC screening rates (V).

- All the elements that should be discussed by GP/nurse to help patients in making informed decisions seemed not to be used (V). Some of these elements seemed to influence patient participation in CRC screening.

- Nurse practitioner/resident training (about CRC screening and communication strategies) and performance communication significantly increased both CRC screening recommendations and completion among patients in general and patients with low literacy skills (VI).

- Community-based interventions such as church-based sessions or in-person interviews significantly increased CRC participation or the intention to be screened in minority subgroups of the US population, especially in the elderly (II).

\section{Recommendations}

10.20 Verbal face-to-face interventions with a nurse or physician could be used to improve knowledge and participation. They would be useful to reach subgroups of the population such as the elderly, minorities and those with low literacy (I-A).

10.21 Nurses and primary care practitioners (GPs) should receive adequate training to be able to help patients in making informed decisions about CRC screening ( $\mathbf{V I}-\mathbf{A})$.

10.22 Community-based verbal face-to-face interventions such as church-based sessions or in-person interviews could be used to reach minorities, in the case where the providers of such interventions received adequate training (II-B).

\subsubsection{Mass media campaigns}

A Cochrane systematic review [43] supports the view that mass media campaigns may have a positive influence upon the way health services are utilised, while the effect on promoting cancer screening is less clear.

Two studies conducted in the late 1980 s combined the free distribution of FOBT kits through pharmacies with repeated educational reports on a local television station $[66,67]$. However, nei- 
ther study included any outcomes addressing the effect advertisements may have had on participation rates or decision-making. A cross-sectional survey [96] aimed at assessing the extent to which mass media campaigns launched since the year 2000 in the USA have achieved the goal of educating the public about CRC and screening. Although the authors concluded that media campaigns can be effective in increasing public awareness about CRC risk, the study was not designed to support this assertion.

Two studies were identified that reported the effect on CRC screening rates after extensive media coverage involving celebrities:

I. In the first study, Brown \& Potosky [13] reported various outcomes related to media coverage of US President Ronald Reagan's CRC episode in July 1985 . The authors reported that there was a transitory increase in public interest in CRC, with a corresponding increase in early detection tests following media coverage of the President's CRC surgery. However, as stated by the authors, the evidence is only suggestive and the methodology of the study quite poor.

II. The second study assessed the impact of a CRC awareness campaign on colonoscopy investigations by a well-known television celebrity [20]. The study found that the awareness campaign was temporally associated with an increase in colonoscopy rates. The authors concluded that a celebrity spokesperson can have a substantial impact on public participation in screening programmes.

Nicholson et al.'s RCT [83] has shown that the way information about colorectal cancer was reported in a medium could influence the motivation to be screened in minority groups: information emphasising the progress African-Americans were making in increasing CRC screening and decreasing CRC mortality led to significantly increase intention to be screened, and counteracted the negative effects of medical mistrust, compared to information emphasising racial disparities.

As media can be a source of information for patients, those in charge of CRC screening programmes should work closely with the media and provide them with up-to-date, accurate and comprehensive information to prevent contradictory, false messages or false expectations being sent to the public.

\section{Summary of evidence}

Several studies have investigated the role that the mass media may have in increasing participation in CRC screening. Unfortunately, the quality of the published studies is quite poor, with the majority failing to include any outcomes assessing the role or effect that advertisements or mass media may have either on the decision-making process or the decision to participate or not in CRC screening.

- Celebrity campaigns were useful to increase participation but the increase was only temporary (V).

- Information emphasising the progress a minority group was making in increasing CRC screening and decreasing CRC mortality led to significantly increase intention to be screened, and counteracted the negative effects of medical mistrust, compared to information emphasising racial disparities (II).

As the media can be a source of information for patients, those in charge of CRC screening programmes should work closely with the media and provide them with up-to-date, accurate and comprehensive information.

\section{Recommendations}

10.23 Mass media campaigns using celebrities may be used to increase the awareness of CRC and CRC screening programmes. However, they should be complemented by other measures as the effects are only temporary $(\mathbf{V}-\mathbf{C})$.

10.24 When addressed to minority groups, information provided by mass media campaigns should emphasise positive progress made by the minority group instead of emphasising racial disparities (VI-C).

10.25 (See below).

\subsubsection{Advocacy groups}

Advocacy groups are playing an increasing role in promoting cancer screening [37]. In colorectal cancer screening, for example, we can refer to the role played by the European Cancer Patient Coalition in the generation of CRC awareness and lobbying for effective CRC screening programmes in Europe. However, there are at present no studies showing the impact of such groups on CRC screening. The role of advocacy groups should be investigated. However, as advocacy groups can be a source of information for patients, e.g. by disseminating education messages to the target audience and providing supportive care during and after treatment patient, screening organisations should share information with advocacy groups to prevent contradictory messages being sent to the public.

\section{Recommendations}

10.25 CRC screening programmes should work closely with advocacy groups and the media and provide them with up-to-date, accurate and comprehensive information about CRC and CRC screening ( $\mathbf{V I}-\mathbf{A})$.

\subsubsection{Communication tools/interventions used to} inform a person of a screening test result and facilitate follow-up of a positive result

In CRC screening, positive results are usually accompanied by information about follow-up. Miglioretti et al. [70] reported that $16 \%$ of patients refused follow-up after a positive FOBT test. A similar figure is reported in many countries worldwide. This result emphasises the need for vigilance and continued effort at patient-centred communication and counselling [121].

Very little is known regarding which interventions should be used to ensure follow-up of patients with abnormal findings in CRC screening. Based on a 2004 systematic review [8], it seems that various interventions such as mail and telephone reminders, telephone counselling, and print educational interventions are effective in increasing follow-up rates of abnormal cancer screening findings. In this review, just four studies were retrieved related to CRC screening. Among these studies, Myers et al.'s RCT (2004) [79] has shown that a reminder-feedback and an educational outreach intervention targeted to the primary care physician were effective in improving follow-up.

A retrospective chart review study [91] has shown that one factor associated with higher rates of colonoscopy after positive FOBT results was the patient having a consultation with a gastroenterologist.

Rubin et al.'s RCT [94] has shown that providing patients with a written copy of their standard colonoscopy screening report at the conclusion of their procedure enhanced recall of the findings and recommendations. 
Zheng et al. [123] investigated the factors relating to adherence to follow-up after an abnormal screening FOBT result. The results of this survey suggest that future interventions should focus on:

- Clarifying misperceptions about follow-up (e.g. understanding the benefits and meanings of follow-up);

- Promoting the acceptance of colonoscopy, as for example patients could perceive unpleasantness regarding preparation for colonoscopy and discomfort of the procedure. Turner et al.'s [110] result supports this finding: a peer coach telephone support, in which former patients who had had a colonoscopy served as peer coaches, scheduled within 2 weeks of the colonoscopy appointment significantly increased screening colonoscopy attendance; and

- Addressing psychological distress (e.g. being afraid of finding cancer), and making follow-up testing more convenient and accessible.

Regarding patient consent, verbal face-to-face intervention before (pre-assessment) and after the endoscopic procedure for programmes undergoing endoscopy (FS or colonoscopy) either for primary screening, or more specifically, as recommended by the EU, for assessment of abnormalities detected in FOBT screening (follow-up): see summary below and Chapter 5 [111] for more details.

\section{Summary of evidence}

- A reminder-feedback and an educational outreach intervention targeted to the primary care physician can be effective in improving follow-up. Providing patients with a written copy of their standard screening report enhanced recall of the findings and recommendations (II).

- Using peer coach telephone support increases colonoscopy attendance: interventions should focus on clarifying misperceptions about follow-up, promoting the acceptance of the followup procedure, addressing psychological distress and making follow-up testing more convenient and accessible (II).

- Obtaining a consultation with a gastroenterologist increases the rates of follow-up colonoscopy (V).

The patient should give consent to the endoscopy procedure and should have the opportunity to withdraw consent at any stage before or during the procedure. Patients should be informed about the outcome of their procedure both orally and with written information before leaving the endoscopy unit. The outcome of screening examinations should be communicated to the primary care doctor or equivalent (see Chapter 5 [111] for more details).

\section{Recommendations}

10.26 A telephone or ideally a verbal face-to-face intervention, e.g. nurse or physician intervention, should be used to inform a patient of a positive screening test result, as obtaining such a result could be a source of psychological distress for the patient. A letter informing the patient should not be used as the only way of notifying a positive result $(\mathrm{VI}-\mathrm{A})$.

10.27 To increase endoscopy follow-up after a positive FOBT and facilitate communication, CRC screening programmes should, where possible:

- Use a reminder-feedback and an educational outreach intervention targeted to the primary care physician (II-A);

- Provide patients with a written copy of their screening report (II-A);

- Facilitate patient consultation with a gastroenterologist (V-B);

- Describe the follow-up procedure, make the follow-up testing more convenient and accessible ( $\mathbf{V I}-\mathbf{A})$; and
- Use direct contact intervention to address psychological distress and other specific barriers (V-B).

From Chapter 5 (see Chapter 5 [111] for more details):

10.28 Each endoscopy service must have a policy for pre-assessment that includes a minimum data set relevant to the procedure. There should be documentation and processes in place to support and monitor the policy (see Ch. 5 [111], Rec. 5.20, Sect 5.3.2) (III-B).

10.29 The endoscopy service must have policies that guide the consent process, including a policy on withdrawal of consent before or during the endoscopic procedure (see Ch. 5 [111], Rec. 5.25, Sect 5.3.1) (VI-B).

10.30 Patients should be informed about the outcome of their procedure before leaving the endoscopy unit and given written information that supports a verbal explanation (see Ch. 5 [111], Rec. 5.26, Sect 5.4.3) (VI-A).

10.31 The outcome of screening examinations should be communicated to the primary care doctor (or equivalent) so that it becomes part of the core patient record (see Ch. 5 [111], Rec. 5.27, Sect 5.5.5) (VI-B).

\subsection{Content that should be included in: the invitation letter and leaflet, the letter and leaflet used to notify results, and the instructions $\nabla$}

\subsubsection{General recommendations}

\section{Summary of evidence}

In organised CRC screening programmes, letters and leaflets are the two most disseminated communication instruments used by health organisations. Letters are generally used to invite people to participate in CRC screening, to notify them of the result of the test and provide information on follow-up. Written materials have advantages such as flexibility of delivery, portability, reusability and can be produced relatively quickly and inexpensively. But they have some obvious limitations: information must be concise, addressed to a general readership and is not effective for individuals who do not read. Leaflets should be used to support and detail the information provided in the letters. Some basic information must be included in the letter in case a person reads only the invitation letter and not the leaflet. Screening programmes should ensure that participants understand the instructions on how to use the FOBT kit and perform the bowel cleansing. Letters, leaflets and written instructions should be developed taking into account all the recommendations given previously.

Currently there is no consensus on what should be said in the letter/leaflet even if the majority of experts agree that individuals must be given information about the pros and the cons of screening to enable IDM. The material listed below could be used as guidelines/examples:

- The recent EU guidelines for cervix cancer screening;

- The IPDAS (an international group of more than 100 researchers, practitioners and stakeholders, see following chapter) recommendations for information content [29];

- The ICSN publication, 2007: "Designing Print Materials: A Communications Guide for Breast Cancer Screening", [81];

- The invitation leaflet developed and used for the UK CRC screening programme (The NHS Bowel Cancer Screening Programme: "Bowel Cancer Screening: the Facts”,

- http://www.cancerscreening.nhs.uk/bowel/publications/ bowel-cancer-the-facts.pdf, and the Evidence Summary: 
patient information for the NHS Bowel cancer screening programme);

- The colonoscopy leaflet developed and used for the UK CRC screening programme (The NHS Bowel Cancer Screening Programme, "Bowel Cancer Screening: The colonoscopy; investigation", http://www.cancerscreening.nhs.uk/bowel/ publications/colonoscopy-investigation.pdf); and/or

- The invitation and colonoscopy leaflets developed and used for the UK CRC screening programme for those with disabilities: http://www.cancerscreening.nhs.uk/bowel/publications/ nhsbcsp-learning-disabilities-leaflet.pdf and http://www.cancerscreening.nhs.uk/bowel/publications/ nhsbcsp-colonoscopy-learning-disabilities-leaflet.pdf

\section{Recommendations}

Letters, leaflets and written instructions (on how to use the FOBT kit and perform the bowel cleansing) should be developed by taking into account all the recommendations below, some of which are either taken from previous relevant sections of Chapter 10 as indicated:

- General principles (Paragraph 10.2): recommendations $10.1-10.5$.

- Physician/GP endorsement, Letters, FOBT delivery and instructions (Paragraph 10.4.1): recommendations 10.6, 10.7, 10.10 .

- Leaflets/booklets (Paragraph 10.4.2.1): recommendations $10.11-10.13$

- Result and follow-up (Paragraph 10.4 .3 and Chapter 5 [111]): $10.27-10.31$.

\section{New recommendations}

10.32 Ideally, the invitation letter and the letter used for notification of a positive result should be sent with a leaflet and participants should be encouraged to read it ( $\mathbf{V I}-\mathbf{A})$.

10.33 Certain basic information e. g. logistic/organisational information, a description of the screening test, the harms and benefits of screening, information about the FOBT kit and the bowel cleansing procedure, must be included in the letter in case a person reads only the invitation/result letter and not the leaflet (VI-A).

\subsubsection{When FOBT is used for screening: content of letters and leaflets}

\subsubsection{FOBT invitation letter}

The letter inviting patients to perform FOBT screening should contain the following information:

\section{- Screening information:}

- The purpose of screening (describe the natural course taken by the disease if not detected and explain the aim of early detection, mention the different prospects depending on whether the disease is found with screening or not, specifically mention the option of not participating);

Who the test is for (target population, age group); and

$\checkmark$ The screening interval.

- Organisational information:

$\checkmark$ How to make and change the appointment when an appointment is required to pick-up the test;

- Cost of the test (free or not); and

- Where further information can be obtained (information services, telephone hotlines, patient groups, websites, etc.).
- Information about the the screening test:

D Details of the screening test that will be performed (including who performs the test, how long it will take, what the test is designed to measure);

- How to obtain the result (mentioning the approximate waiting times); and

- The proportion of people who may require further testing.

- Information about the benefits of screening: Emphasise that early detection can save lives.

- Information about the harms/side effects/disadvantages of screening:

- Meaning of a FOBT positive result in terms of follow-up: what is colonoscopy, benefits and possible harms of the colonoscopy (see Chapter 5 [111] for details), referring to colonoscopy leaflet; and

- Fear/anxiety about cancer and screening results.

- Information about the FOBT kit:

- Where to collect it; and

- If the FOBT kit is sent with the letter, the letter should refer to the instruction leaflet and encourage participants to read it.

- Referral to the invitation leaflet: encouraging participants to read it.

\subsubsection{FOBT invitation leaflet}

The leaflet inviting patients to perform FOBT screening should contain the following information:

\section{- Screening information:}

- The purpose of screening (describe the natural course taken by the disease if not detected and explain the aim of early detection, mention the different prospects depending on whether the disease is found with screening or not, specifically mention the option of not participating)

- Who the test is for (target population, age group);

- The screening interval;

- Quality standards and quality assurance;

- Other types of screening; and

- Comments on people outside the recommended age group, including those at risk of colorectal cancer.

- Colorectal cancer:

Incidence;

Lifetime morbidity and mortality; and

- Risk factors.

- Screening test:

- Nature (what is it?);

- Purpose (what the test is designed to measure);

- Details of the screening test that will be performed (including who performs the test, how long it will take, what the test is designed to measure);

- Informed consent;

- How to obtain the result (mentioning the approximate waiting times);

> Meaning of the test results (What "negative", "positive" and "unclear" mean);

- Meaning of a FOBT positive result in terms of follow-up: what is colonoscopy, benefits and possible harms of the colonoscopy (see Chapter 5 [111] for details), referring to colonoscopy leaflet;

- Mention the proportion of people who may require further testing; and

- Reassurance about follow-up. 


\section{- Test characteristics:}

- False positive and false negative results (including chances of true positive, true negative, false positive, and false negative tests);

- Positive predictive value;

- Number needed to screen to prevent one death; and

$\checkmark$ Reasons why FOBT sometimes need to be repeated.

- Benefits of screening:

- Mention that early detection can save lives;

- Cancer can be found earlier/be prevented; and

- Screening relieves fear and anxiety about cancer; peace of mind.

- Harms/side effects/disadvantages of screening:

- Harms/side effects/disadvantages of colonoscopy if followup is required: sedation, cleansing procedure, possible complications, discomfort and pain during the colonoscopy procedure;

- Identification and treatment of clinically unimportant tumours: the possibility of over-diagnosis; and

- Fear/anxiety about cancer and screening results.

- Options:

- Include deciding on having a colonoscopy or not (describe the natural course taken by the disease if not detected) or being not clear about what to decide (methods for clarifying and expressing values); and

- The opportunity to request to withdraw from the programme.

Guidelines on presenting probabilities of outcomes in an unbiased and understandable way (IPDAS, NHSBSP no. 65, p.5):

- Use event rates specifying the population and time period;

- Compare outcome probabilities using the same denominator, time period, scale;

- Describe uncertainty around probabilities;

- Absolute risk should be used in preference to relative risk;

- Use visual diagrams;

- Use multiple methods to give probabilities (words, numbers, diagrams);

- Allow the patient to select a way of viewing probabilities (words, numbers, diagrams);

- Allow patient to view probabilities based on their own situation (e.g. age); and

- Place probabilities in context of other events.

\subsubsection{FOBT result/follow-up letter}

The letter to inform patients about FOBT screening result should contain the following information:

- The letter should be personalised with the name of the patient and give the FOBT screening test result.

- If the result is negative, its meaning should be explained in terms of the likelihood of having CRC and the possibility of false negatives. The screening interval should be also specified.

- If the test is unclear, its meaning should be explained. If the directives of the screening programme are to repeat the FOBT, the letter should mention it and the patient should be invited to perform a repeat test.

- If the test is positive, its meaning should be explained in terms of the likelihood of having CRC and possibility of false positive. The letter should refer to the colonoscopy leaflet sent with the letter that describes in detail the colonoscopy procedure and should encourage participants to read it. However, certain basic and practical information about the colonoscopy procedure, its harms and benefits, and logistic/organisational infor- mation relating to the colonoscopy appointment must be included in the letter in case a person reads just the letter and not the colonoscopy leaflet.

\subsubsection{Colonoscopy leaflet (see Section 10.5.3.2)}

10.5.3 When flexible sigmoidoscopy (FS) or colonoscopy is used for screening, either as primary screening test (FS or CS) or to follow-up a positive FOBT result (only CS): content of letters and leaflets

\subsubsection{Endoscopy invitation letter}

The letter inviting patients to perform endoscopy screening should contain the following information:

\section{- Screening information:}

- The purpose of screening (describe the natural course taken by the disease if not detected and explain the aim of early detection, mention the different prospects depending on whether the disease is found with screening or not, specifically mention the option of not participating);

- Who the test is for (target population, age group); and

- The screening interval.

- Organisational information:

- How to make and change the appointment;

- Cost of the test (free or not); and

$\checkmark$ Where further information can be obtained (information services, telephone hotlines, patient groups, web sites, etc...).

\section{- Information about the screening test:}

- Details of the screening test that will be performed (including who performs the test, how long it will take, what the test is designed to measure);

- How to obtain the result (mentioning the approximate waiting times); and

- Mention the proportion of people who may require further testing.

- Information about benefits of screening: Early detection can save lives.

- Information about harms/side effects/disadvantages of endoscopy screening (see Chapter 5 [111] for details):

- For both FS (if colonoscopy is used as follow-up procedure) and colonoscopy: The possible complications of colonoscopy and discomfort and pain during the procedure;

- The meaning of a positive FS result in terms of follow-up: what is colonoscopy, benefits and possible harms of the colonoscopy, referring to colonoscopy leaflet; and

- Identification and treatment of clinically unimportant tumours: the possibility of over-diagnosis.

- Information about the cleansing procedure.

- Referral to the endoscopy leaflet encouraging participants to read it.

- Options:

- Include deciding whether to have an endoscopy (describe the natural course without having the endoscopy), or being not clear about what to decide (methods for clarifying and expressing values); and

- The possibility to withdraw consent at any stage (Chapter 5 [111] recommendation). 


\subsubsection{Endoscopy invitation leaflet: example for} colonoscopy

The leaflet to inform patients about a colonoscopy screening, either for primary screening or as follow-up after a positive FOBT or FS, should contain the following information:

- Colorectal cancer and colorectal screening:

- The purpose and the importance of screening; what early detection means;

$\checkmark$ A description of colorectal cancer disease; and

- General information about the CRC screening programme.

- In cases where colonoscopy is used as follow-up after a positive FOBT result or FS:

- Explain why colonoscopy is required;

- How to interpret a FS positive result; and

- How to interpret a FOBT positive result: What "positive FOBT" result means: including chances of true positive, true negative, false positive and false negative test.

- Colonoscopy procedure:

Nature (what is it?);

- Who the test is for; validity;

- Purpose (what the test is designed to measure, why it is being done);

- How to make and change an appointment;

- How the test is carried out;

- How to prepare for the colonoscopy (including bowel cleansing and options for sedation);

- Who performs the test, where it is performed;

- How long it takes;

- What to do when the test is done;

- Cost of the procedure: free or not;

- How to obtain the result (approximate waiting times);

- Meaning of colonoscopy results (normal, polyps, cancer);

- Quality control of the colonoscopy procedure; and

- What to do if people have symptoms after colonoscopy.

- Positive outcomes: Cancers can be found earlier/be prevented.

- Harms/side effects/disadvantages of colonoscopy

(see Chapter 5 [111] for details):

- Associated restrictions on travelling or making important decisions due to sedation;

$\checkmark$ Cleansing procedure;

- Possible adverse events including discomfort, pain and complications;

- Identification and treatment of clinically unimportant tumours: the possibility of over-diagnosis;

- Fear/anxiety about cancer and colonoscopy results; and

- What support may be needed after the procedure, particularly if the patient is sedated.

Options:

- Include deciding on having a colonoscopy or not (describe the natural course without having the colonoscopy), or being not clear about what to decide (methods for clarifying and expressing values)

- The opportunity to withdraw consent at any stage (Chapter 5 [111] recommendation)

Guidelines on how to present probabilities of outcomes in an unbiased and understandable way (IPDAS, NHSBSP no65 p5) as described above for the invitation leaflet.
- If the result is negative, its meaning should be explained in terms of the likelihood of having CRC and possibility of false negatives. The screening interval should be also specified;

- If the test is positive, the letter should describe in detail what following steps to take.

\subsection{Stylistic advice}

\section{$\nabla$}

The way information is presented plays an important role in determining its comprehension and acceptance. For this reason, it is essential that written information be guided by good communication principles in order to be easy to read and understood by the users.

Written information material should be clear, visually appealing and motivating to the intended audience.

Some recommendations on language, on text style and wording, and formatting are provided hereafter, based on the recent EU guidelines for quality assurance in cervical cancer screening [2]. They should be carefully considered by the screening staff to make the communication more effective and easily understandable to participants.

\section{Recommendations}

The language, text style, wording and formatting used in written information should follow these suggestions:

- Language:

- Clear (about the topic: clarify points with examples);

- Honest, respectful, polite;

- Simple everyday language (no technical terms, jargon, abbreviations and acronyms);

> Informal (use of pronouns like "we" and "you" to personalise the text);

- Impartial;

- Not top-down (no prescriptive style or paternalistic tone); and

Written in the active voice.

- Text style and wording:

- Credible, reliable (indicating the source of information);

- Up-to-date and contemporary;

$\checkmark$ Friendly and sympathetic;

- Positively framed (e.g. 9 out of 10 recalled patients are found to be normal rather than 1 out of 10 recalled women will have cancer); and

- Positive tone (alarming statements should be avoided).

- Text format:

- Preferably plain layout;

- Short sentences and brief paragraphs;

- Use of diagrams and pictures;

- Use of titles and subtitles (to distinguish different areas);

- Bold or capital letters (to underline important points);

- Larger print (essential for older target populations);

- Use of white spaces (to facilitate reading);

- Preferably question/answer and paragraph formats;

- Appropriate colours (as some colours are difficult for colour-blind people to read); and

$>$ Logo.

\subsubsection{Endoscopy results/follow-up letter}

The letter should be personalised with the name of the patient and give the endoscopy screening test result: 


\subsection{Evaluating the quality of public information materials: are these materials meeting the required standard for quality? \\ $\nabla$}

There are currently different guides to assess the quality of communications tools. The International Patient Decision Aid Standard (IPDAS) collaboration group (an international group of more than 100 researchers, practitioners and stakeholders) has provided a framework of quality criteria for patient decisions aids used for screening or health decisions [29]. Even if the IPDAS checklist does not address CRC screening specifically, it is a good guideline for evaluating the quality of communication tools produced by CRC screening programmes. This is the reason why we recommend using it.

The IPDAS framework, a list of 80 items, was produced as a consensus of the IPDAS group and developed based on evidence where it exists and the view of IPDAS experts. These criteria "might be considered to represent an ideal construction that may be difficult to attain. The criteria are not meant to be prescriptive." [29]. The criteria (in Developing a quality criteria framework for patient decision aids: online international Delphi consensus process and IPDAS criteria checklist) address 3 domains of quality: the content (specific to the health condition and therapeutic/screening options), the development process (referring to the way the decision aid should be developed and relevant to any decision aid) and the effectiveness (relevant to any decision aid, to evaluate the effectiveness of the decision aid). Based on these criteria, a new instrument has been developed to assess the quality of decision support materials: the IPDASi assessment service (http://www.ipdasi.org/) which is currently undertaking a validation study assessing 30 decision support technologies.

\section{Conclusions \\ $\nabla$}

In a multidisciplinary process, wide consensus has been achieved on a comprehensive package of evidence-based recommendations for quality assurance in communication in colorectal cancer screening. Following these recommendations has the potential to enhance the control of colorectal cancer in Europe and elsewhere through improvement in the quality and effectiveness of the screening process that extends from systematic invitation to management of screen-detected cases.

\section{Disclaimer \\ $\nabla$}

The views expressed in this document are those of the authors. Neither the European Commission nor any person acting on its behalf can be held responsible for any use that may be made of the information in this document.

Competing interests: No competing interests reported.

\section{Acknowledgements \\ $\nabla$}

The comments and suggestions received from the following reviewer are gratefully acknowledged: Berta Geller, United States of America.

The comments and suggestions received from consultation of the European Cancer Network are gratefully acknowledged.
The production of the Guidelines was supported by the European Union through the EU Public Health Programme, (grant agreement no.2005317: Development of European Guidelines for Quality Assurance of Colorectal Cancer Screening). Partner institutions: Oxford University Cancer Screening Research Unit, Cancer Epidemiology Unit, University of Oxford, Oxford, United Kingdom; Unit of Cancer Epidemiology, Centre for Cancer Epidemiology and Prevention (CPO) and S. Giovanni University Hospital, Turin, Italy; Public Association for Healthy People, Budapest, Hungary; European Cancer Patient Coalition (ECPC), Utrecht, Netherlands ; Quality Assurance Group, Section of Early Detection and Prevention, International Agency for Research on Cancer, Lyon, France.

Financial support was also received through the Public Affairs Committee of the United European Gastroenterology Federation, and from a cooperative agreement between the American Cancer Society and the Division of Cancer Prevention and Control at the Centers for Disease Control and Prevention.

\section{References}

1 Abbott PA, Coenen A. Globalization and advances in information and communication technologies: the impact on nursing and health. Nurs Outlook 2008; 56: 238-246

2 Arbyn M, Anttila A, Jordan J et al. (eds.) European guidelines for quality assurance in cervical cancer screening - second edition. 2nd: edition Luxembourg: Office for Official Publications of the European Communities; 2008

3 Aro AR, de Koning HJ, Absetz P et al. Psychosocial predictors of first attendance for organised mammography screening. J Med Screen 1999; 6: $82-88$

4 Atkin W, Valori R, Kuipers EJ et al. European guidelines for quality assurance in colorectal cancer screening and diagnosis. First Edition. Colonoscopic surveillance following adenoma removal. Endoscopy 2012

5 Austoker J. Gaining informed consent for screening. Is difficult - but many misconceptions need to be undone.. BMJ 1999; 319: 722 -723

6 Baker DW, Gazmararian JA, Sudano J et al. Health literacy and performance on the Mini-Mental State Examination. Aging Ment Health 2002; 6: $22-29$

7 Basch CE, Wolf RL, Brouse CH et al. Telephone outreach to increase colorectal cancer screening in an urban minority population. Am J Public Health 2006; 96: 2246-2253

8 Bastani R, Yabroff KR, Myers RE et al. Interventions to improve followup of abnormal findings in cancer screening. Cancer 2004; 101: $1188-$ 1200

9 Beauchamp TL, Childress G. Principles of Biomedical Ethics. Oxford: Oxford University; 1979

10 Braun KL, Fong $M$, Kaanoi ME et al. Testing a culturally appropriate, theory-based intervention to improve colorectal cancer screening among Native Hawaiians. Prev Med 2005; 40: 619-627

11 Brodie $M$, Foehr $U$, Rideout $V$ et al. Communicating health information through the entertainment media. Health Aff (Millwood.) 2001; 20: $192-199$

12 Brotherstone $H$, Miles A, Robb KA et al. The impact of illustrations on public understanding of the aim of cancer screening. Patient Educ Couns 2006; 63: $328-335$

13 Brown ML, Potosky AL. The presidential effect: the public health response to media coverage about Ronald Reagan's colon cancer episode. Public Opin Q 1990; 54: 317 - 329

14 Campbell MK, James A, Hudson MA et al. Improving multiple behaviors for colorectal cancer prevention among african american church members. Health Psychol 2004; 23: 492-502

15 Chan EC, Vernon SW. Implementing an intervention to promote colon cancer screening through e-mail over the Internet: lessons learned from a pilot study. Med Care 2008; 46: S117-S122

16 Christie J, Itzkowitz S, Lihau-Nkanza I et al. A randomized controlled trial using patient navigation to increase colonoscopy screening among low-income minorities. J Natl Med Assoc 2008; 100: 278-284

17 Costanza ME, Luckmann R, Stoddard AM et al. Using tailored telephone counseling to accelerate the adoption of colorectal cancer screening. Cancer Detect Prev 2007; 31: $191-198$ 
18 Council of the European Union. Council Recommendation of 2 December 2003 on cancer screening (2003/878/EC). Off J Eur Union; 2003: $34-38$

19 Courtier R, Casamitjana M, Macia F et al. Participation in a colorectal cancer screening programme: influence of the method of contacting the target population. Eur J Cancer Prev 2002; 11: 209-213

20 Cram P, Fendrick AM, Inadomi J et al. The impact of a celebrity promotional campaign on the use of colon cancer screening: the Katie Couric effect. Arch Intern Med 2003; 163: 1601 - 1605

21 Davis TC, Williams MV, Marin E et al. Health literacy and cancer communication. CA Cancer J Clin 2002; 52: 134-149

22 Della LJ, Eroglu D, Bernhardt JM et al. Looking to the future of new media in health marketing: deriving propositions based on traditional theories. Health Mark Q 2008; 25: 147-174

23 Denberg TD, Coombes JM, Byers TE et al. Effect of a mailed brochure on appointment-keeping for screening colonoscopy: a randomized trial. Ann Intern Med 2006; 145: 895-900

24 Dewalt DA, Berkman ND, Sheridan S et al. Literacy and health outcomes: a systematic review of the literature. J Gen Intern Med 2004; 19: $1228-1239$

25 Dexheimer JW, Talbot TR, Sanders DL et al. Prompting clinicians about preventive care measures: a systematic review of randomized controlled trials. J Am Med Inform Assoc 2008; 15: 311 - 320

26 Dietrich AJ, Tobin JN, Cassells A et al. Telephone care management to improve cancer screening among low-income women: a randomized, controlled trial. Ann Intern Med 2006; 144: 563-571

27 Dolan JG, Frisina S. Randomized controlled trial of a patient decision aid for colorectal cancer screening. Med Decis Making 2002; 22: $125-139$

28 Dolan NC, Ferreira MR, Davis TC et al. Colorectal cancer screening knowledge, attitudes, and beliefs among veterans: does literacy make a difference? J Clin Oncol 2004; 22: 2617-2622

29 Elwyn G, O'Connor A, Stacey $D$ et al. Developing a quality criteria framework for patient decision aids: online international Delphi consensus process. BMJ 2006; 333: 417

30 Ferlay J, Shin HR, Bray F et al. GLOBOCAN 2008 v1.2, Cancer Incidence and Mortality Worldwide: IARC CancerBase No. 10. [Internet] Lyon, France: International Agency for Research on Cancer; 2010: Available from: http://globocan.iarc.fr Accessed on 05/04/2012

31 Ferreira MR, Dolan NC, Fitzgibbon ML et al. Health care provider-directed intervention to increase colorectal cancer screening among veterans: results of a randomized controlled trial. J Clin Oncol 2005; 23: $1548-1554$

32 Ford ME, Havstad SL, Davis SD. A randomized trial of recruitment methods for older African American men in the Prostate, Lung, Colorectal and Ovarian (PLCO) Cancer Screening Trial. Clin Trials 2004; 1 : $343-351$

33 Fox $R$. Informed choice in screening programmes: do leaflets help? A critical literature review. J Public Health (Oxf) 2006; 28: 309-317

34 Fox S, Rainie $L$. The on-line health care revolution: How the Web helps Americans take better care of themselves. Washington D.C: The Pew Internet \& American Life Project; 2000

35 Friedemann-Sanchez G, Griffin JM, Partin MR. Gender differences in colorectal cancer screening barriers and information needs. Health Expect 2007; 10: $148-160$

36 Friedman LC, Everett TE, Peterson $L$ et al. Compliance with fecal occult blood test screening among low-income medical outpatients: a randomized controlled trial using a videotaped intervention. J Cancer Educ 2001; 16: 85-88

37 Ganz PA. Advocating for the woman with breast cancer. CA Cancer J Clin 1995; 45: 114-126

38 Geller BM, Skelly JM, Dorwaldt AL et al. Increasing patient/physician communications about colorectal cancer screening in rural primary care practices. Med Care 2008; 46: 36-43

39 Giordano L, Webster P, Anthony C et al. Improving the quality of communication in organised cervical cancer screening programmes. Patient Educ Couns 2008; 72: 130-136

40 Goyder E, Barratt A, Irwig LM. Telling people about screening programmes and screening test results: how can we do it better? J Med Screen 2000; 7: 123-126

41 Gren L, Broski K, Childs J et al. Recruitment methods employed in the Prostate, Lung, Colorectal, and Ovarian Cancer Screening Trial. Clin Trials 2009; 6: $52-59$
42 Griffith JM, Lewis CL, Brenner AR et al. The effect of offering different numbers of colorectal cancer screening test options in a decision aid: a pilot randomized trial. BMC Med Inform Decis Mak 2008; 8: 4

43 Grilli R, Ramsay C, Minozzi S. Mass media interventions: effects on health services utilisation. Cochrane Database Syst Rev 2002: CD000389

44 Halloran S, Launoy G, Zappa M. European guidelines for quality assurance in colorectal cancer screening and diagnosis. First Edition. Faecal Occult Blood Testing. Endoscopy 2012

45 Hart AR, Barone TL, Gay SP et al. The effect on compliance of a health education leaflet in colorectal cancer screening in general practice in central England. J Epidemiol Community Health 1997; 51: 187-191

46 Holland W, Stewart S, Masseria C. Policy Brief: Screening in Europe. Copenhagen: WHO Regional Office; 2006

47 Hudson SV, Ohman-Strickland P, Cunningham $R$ et al. The effects of teamwork and system support on colorectal cancer screening in primary care practices. Cancer Detect Prev 2007; 31: 417-423

48 James $N$, Daniels $H$, Rahman $R$ et al. A study of information seeking by cancer patients and their carers. Clin Oncol (R Coll Radiol) 2007; 19: $356-362$

49 Jandorf L, Gutierrez Y, Lopez J et al. Use of a patient navigator to increase colorectal cancer screening in an urban neighborhood health clinic. J Urban Health 2005; 82: 216-224

50 Jepson RG, Hewison J, Thompson AG et al. How should we measure informed choice? The case of cancer screening. J Med Ethics 2005; 31: $192-196$

51 Jerant A, Kravitz RL, Rooney $M$ et al. Effects of a tailored interactive multimedia computer program on determinants of colorectal cancer screening: a randomized controlled pilot study in physician offices. Patient Educ Couns 2007; 66: $67-74$

52 Jimbo M, Nease DEJr, Ruffin MT et al. Information technology and cancer prevention. CA Cancer J Clin 2006; 56: 26-36

$53 \mathrm{Katz}$ ML, Tatum C, Dickinson SL et al. Improving colorectal cancer screening by using community volunteers: results of the Carolinas cancer education and screening (CARES) project. Cancer 2007; 110 : $1602-1610$

54 Kim J, Whitney A, Hayter S et al. Development and initial testing of a computer-based patient decision aid to promote colorectal cancer screening for primary care practice. BMC Med Inform Decis Mak 2005; 5: 36

55 Lagerlund M, Sparen P, Thurfjell E et al. Predictors of non-attendance in a population-based mammography screening programme; socio-demographic factors and aspects of health behaviour. Eur J Cancer Prev 2000; 9: 25-33

56 Lairson DR, Dicarlo M, Myers RE et al. Cost-effectiveness of targeted and tailored interventions on colorectal cancer screening use. Cancer 2008; $112: 779-788$

57 Landsorp-Vogelaar I, von Karsa L. European guidelines for quality assurance in colorectal cancer screening and diagnosis. First Edition. Introduction. Endoscopy 2012

58 Lasser KE, Ayanian JZ, Fletcher RH et al. Barriers to colorectal cancer screening in community health centers: a qualitative study. BMC Fam Pract 2008; 9: 15

59 Lewis CL, Brenner AT, Griffith JM et al. The uptake and effect of a mailed multi-modal colon cancer screening intervention: A pilot controlled trial. Implement Sci 2008; 3: 32

60 Ling BS, Trauth JM, Fine MJ et al. Informed decision-making and colorectal cancer screening: is it occurring in primary care? Med Care 2008; 46: 23-S29

61 Lipkus IM, Green LG, Marcus A. Manipulating perceptions of colorectal cancer threat: implications for screening intentions and behaviors. J Health Commun 2003; 8: 213-228

62 Lipkus IM, Skinner CS, Dement J et al. Increasing colorectal cancer screening among individuals in the carpentry trade: test of risk communication interventions. Prev Med 2005; 40: 489-501

63 Makoul G, Cameron KA, Baker DW et al. A multimedia patient education program on colorectal cancer screening increases knowledge and willingness to consider screening among Hispanic/Latino patients. Patient Educ Couns 2009; 76: 220 - 226

64 Malila N, Senore C, Armaroli P. European guidelines for quality assurance in colorectal cancer screening and diagnosis. First Edition. Organisation. Endoscopy 2012

65 Marcus AC, Mason M, Wolfe P et al. The efficacy of tailored print materials in promoting colorectal cancer screening: results from a random- 
ized trial involving callers to the National Cancer Institute's Cancer Information Service. J Health Commun 2005; 10: 83-104

66 McGarrity TJ, Long PA, Peiffer LP. Results of a repeat television-advertised mass screening program for colorectal cancer using fecal occult blood tests. Am J Gastroenterol 1990; 85: 266-270

67 McGarrity TJ, Long PA, Peiffer LP et al. Results of a television-advertised public screening program for colorectal cancer. Arch Intern Med 1989; 149: $140-144$

68 Meade CD, McKinney WP, Barnas GP. Educating patients with limited literacy skills: the effectiveness of printed and videotaped materials about colon cancer. Am J Public Health 1994; 84: 119-121

69 Menon $U$, Szalacha $L A$, Belue $R$ et al. Interactive, culturally sensitive education on colorectal cancer screening. Med Care 2008; 46: 44-50

70 Miglioretti DL, Rutter CM, Bradford SC et al. Improvement in the diagnostic evaluation of a positive fecal occult blood test in an integrated health care organization. Med Care 2008; 46: 91 -S96

71 Miller DPJr, Kimberly JRJr, Case LD et al. Using a computer to teach patients about fecal occult blood screening. A randomized trial. J Gen Intern Med 2005; 20: 984-988

72 Minozzi S, Armaroli P, Banzi R et al. European guidelines for quality assurance in colorectal cancer screening and diagnosis - First edition. Appendix 1: Systematic evidence review. 2010: http://bookshop. europa.eu/en/european-guidelines-for-quality-assurance-incolorectal-cancer-screening-and-diagnosis-pbND3210390/ Accessed $11 / 2 / 2012$

73 Minozzi S, Armaroli P, Segnan N. European guidelines for quality assurance in colorectal cancer screening and diagnosis. First Edition. Principles of evidence assessment and methods for reaching recommendations. Endoscopy 2012

74 Moss S, Ancelle-Park R, Brenner H. European guidelines for quality assurance in colorectal cancer screening and diagnosis. First Edition. Evaluation and interpretation of screening outcomes. Endoscopy 2012

75 Myers RE, Hyslop T, Sifri R et al. Tailored navigation in colorectal cancer screening. Med Care 2008; 46: 123-131

76 Myers RE, Ross E, Jepson C et al. Modeling adherence to colorectal cancer screening. Prev Med 1994; 23: $142-151$

77 Myers RE, Ross EA, Wolf TA et al. Behavioral interventions to increase adherence in colorectal cancer screening. Med Care 1991; 29: 10391050

78 Myers RE, Sifri R, Hyslop T et al. A randomized controlled trial of the impact of targeted and tailored interventions on colorectal cancer screening. Cancer 2007; 110: 2083 -2091

79 Myers RE, Turner B, Weinberg $D$ et al. Impact of a physician-oriented intervention on follow-up in colorectal cancer screening. Prev Med 2004; 38: 375-381

80 Nash D, Azeez S, Vlahov D et al. Evaluation of an intervention to increase screening colonoscopy in an urban public hospital setting. J Urban Health 2006; 83: 231 - 243

81 National Cancer Institute (NCI). Designing Print Materials: A communications guide for breast cancer screening. Bethesda: International Cancer Screening Network; 2007: http://appliedresearch.cancer.gov/ icsn/publications/designing_print_materials_bw.pdf Accessed 11/2/ 2012

82 Nease DEJr, Ruffin MT, Klinkman MS et al. Impact of a generalizable reminder system on colorectal cancer screening in diverse primary care practices: a report from the prompting and reminding at encounters for prevention project. Med Care 2008; 46: $68-73$

83 Nicholson RA, Kreuter MW, Lapka C et al. Unintended effects of emphasizing disparities in cancer communication to African-Americans. Cancer Epidemiol Biomarkers Prev 2008; 17: 2946-2953

84 O'Connor AM, Bennett CL, Stacey D et al. Decision aids for people facing health treatment or screening decisions. Cochrane Database Syst Rev 2009: CD001431

85 Percac-Lima S, Grant RW, Green AR et al. A culturally tailored navigator program for colorectal cancer screening in a community health center: a randomized, controlled trial. J Gen Intern Med 2009; 24: 211-217

86 Pereira JL, Koski S, Hanson J et al. Internet usage among women with breast cancer: an exploratory study. Clin Breast Cancer 2000; 1: 148 153

87 Pignone $M$, Harris $R$, Kinsinger $L$. Videotape-based decision aid for colon cancer screening. A randomized, controlled trial. Ann Intern Med 2000; 133: 761 - 769

88 Powe BD, Ntekop E, Barron M. An intervention study to increase colorectal cancer knowledge and screening among community elders. Public Health Nurs 2004; 21: 435-442
89 Quirke P, Risio M, Lambert R et al. European guidelines for quality assurance in colorectal cancer screening and diagnosis. First Edition. Quality assurance in pathology in colorectal cancer screening and diagnosis. Endoscopy 2012

90 Raffle AE. Informed participation in screening is essential. BMJ 1997; 314: $1762-1763$

91 Rao SK, Schilling TF, Sequist TD. Challenges in the management of positive fecal occult blood tests. J Gen Intern Med 2009; 24: 356-360

92 Rimer BK, Briss PA, Zeller PK et al. Informed decision making: what is its role in cancer screening? Cancer 2004; 101: 1214-1228

93 Robb KA, Miles A, Campbell J et al. Can cancer risk information raise awareness without increasing anxiety? A randomized trial. Prev Med 2006; 43: 187-190

94 Rubin DT, Ulitsky A, Poston J et al. What is the most effective way to communicate results after endoscopy? Gastrointest Endosc 2007; 66: $108-112$

95 Ruffin MT, Fetters MD, Jimbo M. Preference-based electronic decision aid to promote colorectal cancer screening: results of a randomized controlled trial. Prev Med 2007; 45: 267-273

96 Schroy PCIII, GlickJT, Robinson PA et al. Has the surge in media attention increased public awareness about colorectal cancer and screening? J Community Health 2008; 33: 1 -9

97 Segnan N, Patnick J, von Karsa L et al. European guidelines for quality assurance in colorectal cancer screening and diagnosis - First edition. Luxembourg: European Commission, Publications Office of the European Union; 2010

98 Senore C, Armaroli P, Silvani $M$ et al. Comparing different strategies for colorectal cancer screening in Italy: predictors of patients' participation. Am J Gastroenterol 2010; 105: 188 - 198

99 Sequist TD, Zaslavsky AM, Marshall R et al. Patient and physician reminders to promote colorectal cancer screening: a randomized controlled trial. Arch Intern Med 2009; 169: 364-371

100 Department for Education and Skills. Skills for life survey. A national needs and impact survey of literacy, numeracy and ITC skills. 2003: http://www.dcsf.gov.uk/research/data/uploadfiles/RB490.pdf Accessed $11 / 2 / 2012$

101 Steckelberg A, Kasper J, Redegeld $M$ et al. Risk information-barrier to informed choice? A focus group study. Soz Praventivmed 2004; 49: $375-380$

102 Steele RJC, Pox C, Kuipers EJ et al. European guidelines for quality assurance in colorectal cancer screening and diagnosis. First Edition. Managment of lesions detected in colorectal cancer screening. Endoscopy 2012

103 Steele RJC, Rey J-F, Lambert R. European guidelines for quality assurance in colorectal cancer screening and diagnosis: First edition. Professional requirements and training. Endoscopy 2012; 44: SE106SE115

104 Stokamer CL, Tenner CT, Chaudhuri J et al. Randomized controlled trial of the impact of intensive patient education on compliance with fecal occult blood testing. J Gen Intern Med 2005; 20: 278 - 282

105 Sudore RL, Mehta KM, Simonsick EM et al. Limited literacy in older people and disparities in health and healthcare access. J Am Geriatr Soc 2006; 54: 770-776

106 Sudore RL, Yaffe K, Satterfield S et al. Limited literacy and mortality in the elderly: the health, aging, and body composition study. J Gen Intern Med 2006; 21: 806-812

107 Thompson NJ, Boyko EJ, Dominitz JA et al. A randomized controlled trial of a clinic-based support staff intervention to increase the rate of fecal occult blood test ordering. Prev Med 2000; 30: 244-251

108 Tilley BC, Vernon SW, Myers $R$ et al. The Next Step Trial: impact of a worksite colorectal cancer screening promotion program. Prev Med 1999; 28: 276-283

109 Trevena LJ, Irwig L, Barratt A. Randomized trial of a self-administered decision aid for colorectal cancer screening. J Med Screen 2008; 15 : $76-82$

110 Turner BJ, Weiner M, Berry SD et al. Overcoming poor attendance to first scheduled colonoscopy: a randomized trial of peer coach or brochure support. J Gen Intern Med 2008; 23: 58-63

111 Valori R, Rey J-F, Atkin $W$ et al. European guidelines for quality assurance in colorectal cancer screening and diagnosis. First Edition. Quality assurance in endoscopy in colorectal screening and diagnosis. Endoscopy 2012

112 van Rijn AF, van Rossum LG, Deutekom M et al. Getting adequate information across to colorectal cancer screening subjects can be difficult. J Med Screen 2008; 15: 149-152 
113 van Wieringen JC, Harmsen JA, Bruijnzeels MA. Intercultural communication in general practice. Eur J Public Health 2002; 12: 63-68

114 Vernon SW, Meissner HI. Evaluating approaches to increase uptake of colorectal cancer screening: lessons learned from pilot studies in diverse primary care settings. Med Care 2008; 46: 97-102

115 von Karsa L, Anttila A, Ronco G et al. Cancer Screening in the European Union. Report on the implementation of the Council Recommendation on Cancer Screening - First Report. Luxembourg: European Commission; 2008: http://ec.europa.eu/health/archive/ph_determinants/ genetics/documents/cancer_screening.pdf Accessed 11/2/2012

116 von Karsa L, Lignini TA, Patnick J et al. The dimensions of the CRC problem. Best Pract Res Clin Gastroenterol 2010; 24: 381 - 396

117 Wardle J, Williamson S, McCaffery $K$ et al. Increasing attendance at colorectal cancer screening: testing the efficacy of a mailed, psychoeducational intervention in a community sample of older adults. Health Psychol 2003; 22: 99-105
118 Wee CC, McCarthy EP, Phillips RS. Factors associated with colon cancer screening: the role of patient factors and physician counseling. Prev Med 2005; 41: 23-29

119 Wilson JM, Jungner YG. Principles and practice of mass screening for disease. World Health Organization; 1968: http://whqlibdoc.who. int/php/WHO_PHP_34.pdf Accessed 11/2/2012

120 Woodrow C, Watson E, Rozmovits $L$ et al. Public perceptions of communicating information about bowel cancer screening. Health Expect 2008; $11: 16-25$

121 Zapka J. Innovative provider- and health system-directed approaches to improving colorectal cancer screening delivery. Med Care 2008; 46: $62-S 67$

122 Zapka JG, Lemon SC, Puleo E et al. Patient education for colon cancer screening: a randomized trial of a video mailed before a physical examination. Ann Intern Med 2004; 141: 683-692

123 Zheng YF, Saito T, Takahashi M et al. Factors associated with intentions to adhere to colorectal cancer screening follow-up exams. BMC Public Health 2006; 6: 272 